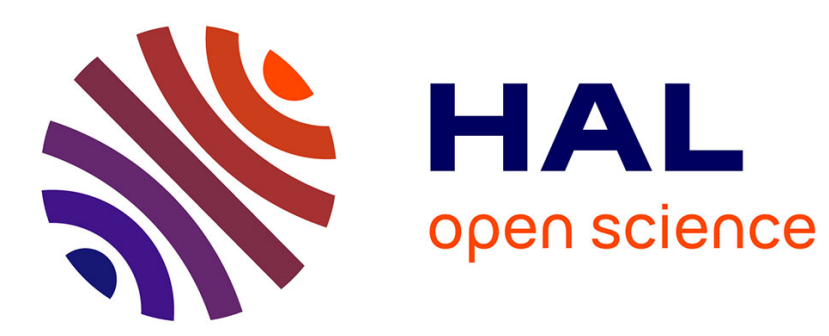

\title{
Idempotent Block Splitting on Partial Partitions, II: Non-isotone Operators
}

Christian Ronse

\section{To cite this version:}

Christian Ronse. Idempotent Block Splitting on Partial Partitions, II: Non-isotone Operators. Order, 2011, 28 (2), pp.307-339. 10.1007/s11083-010-9190-0 . hal-02882872

\section{HAL Id: hal-02882872}

\section{https://hal.science/hal-02882872}

Submitted on 6 Oct 2020

HAL is a multi-disciplinary open access archive for the deposit and dissemination of scientific research documents, whether they are published or not. The documents may come from teaching and research institutions in France or abroad, or from public or private research centers.
L'archive ouverte pluridisciplinaire HAL, est destinée au dépôt et à la diffusion de documents scientifiques de niveau recherche, publiés ou non, émanant des établissements d'enseignement et de recherche français ou étrangers, des laboratoires publics ou privés. 


\title{
Idempotent block splitting on partial partitions, II: non-isotone operators
}

\author{
Christian Ronse
}

Received: date / Revised: date

\begin{abstract}
Our first paper introduced block splitting operators on the complete lattice of partial partitions, studied their algebraic properties and characterized block splitting openings (kernel operators) in terms of partial connections.

In this second paper we study non-isotone idempotent block splitting operators on partial partitions. In particular we analyse the following two constructions:

- the residual combination of block splitting openings, where the $(n+1)$-th opening is applied to the "residue" of the $n$-th one;

- a supremum of operators obtained by composition of a block splitting opening followed by a block selection operator guided by a predicate on sets.

These operators belong to two families of idempotent operators generalizing openings that the author studied previously.

In the same way as the openings analysed in the first paper, these two types of idempotent operators underlie recent image segmentation approaches due to Serra and Soille.
\end{abstract}

Keywords partial partitions · complete lattice · block splitting · idempotence $\cdot$ image segmentation

Mathematics Subject Classification (2000) 03E02 - 06B99 • 06A15 • $68 \mathrm{U} 10$

\section{Introduction}

This is the second part of a general study of block splitting operators on partial partitions, and conditions for their idempotence. Our basic motivation

C. Ronse

LSIIT UMR 7005 CNRS-UdS,

Parc d'Innovation, Boulevard Sébastien Brant, BP 10413, 67412 ILLKIRCH CEDEX, FRANCE

E-mail: cronse@unistra.fr, URL: http://siit-miv.u-strasbg.fr/ 
is the mathematical analysis of the operations involved in some recent image segmentation approaches $[22,23,28]$.

In the first paper, we have analysed the general lattice-theoretical and monoid properties of block splitting operators. Then we showed that block splitting openings coincide with the decomposition of blocks into their connected components according to a partial connection, and that they constitute a complete sublattice of the complete lattice of all openings on partial partitions. These results underlie the connective model for image segmentation $[23,17,13]$ : given $E$ the space of points and $T$ the set of image values, a criterion cr: $T^{E} \times \mathcal{P}(E) \rightarrow\{0,1\}$ associates to an image $F: E \rightarrow T$ and a set $A \in \mathcal{P}(E)$ the value $\operatorname{cr}[F, A]=1$ if $F$ is homogeneous on $A$ according to $\mathrm{cr}$, and $\operatorname{cr}[F, A]=0$ if not; when for every $F: E \rightarrow T$, the set $\mathcal{C}_{\mathrm{cr}}^{F}$ of all $A \in \mathcal{P}(E)$ such that $\operatorname{cr}[F, A]=1$ is a partial connection, the criterion $\mathrm{cr}$ is said to be partially connective, and then for every $A \in \mathcal{P}(E)$ the segmentation of $F$ on $A$ is given by the partial partition $\mathrm{PC}^{\mathcal{C}_{\mathrm{cr}}^{F}}(A)$ of all $\mathcal{C}_{\mathrm{cr}}^{F}$-components of $A$. Similarly, omitting images $E \rightarrow T$, one can decompose shapes (subsets of $E$ ) by using a partial connection $\mathcal{C}$ : every object $A \in \mathcal{P}(E)$ will be decomposed into the partial partition of its $\mathcal{C}$-components.

However in many practical situations, the underlying principle that "given overlapping image regions satisfying the criterion, their union will satisfy that criterion", does not give optimal results. We give here three examples (in the digital plane $E=\mathbf{Z}^{2}$ ) of shape decomposition that do not follow this approach, they correspond in fact to the three set splitting methods studied in Section 3.

Let us first consider a set splitting operator $\sigma$ extracting narrow lines in subsets of $E$. A line segment $A$ is indeed narrow, so from $A$ one extracts $A$ itself; now a square $B$ containing $A$ is not narrow, so from $B$ nothing is extracted. Formally, $\sigma$ satisfies $\sigma(A)=\mathbf{1}_{A}=\{A\}$ and $\sigma(B)=\emptyset$, but $A \subseteq B$ and $\mathbf{1}_{A}>\varnothing$, thus $\sigma$ is not isotone. The design of such an operator $\sigma$ can involve non-isotone template matching operators devised for processing sets or greylevel images $[2,8,9]$, followed by a partitioning into connected components, see Proposition 8 and Figure 4.

Next, we remark that a block splitting opening that extracts from a set two or more types of objects, cannot separate two objects of distinct types when they overlap. This is illustrated in Figure 1 for horizontal and vertical objects. The solution is to apply to a set $A$ a first set splitting operator $\sigma_{1}$ extracting the first type of objects, then to apply to the residual $A \backslash \operatorname{supp}\left[\sigma_{1}(A)\right]$ a second set splitting operator $\sigma_{2}$, and take the compound partial partition $\sigma_{1}(A) \cup \sigma_{2}\left(A \backslash \operatorname{supp}\left[\sigma_{1}(A)\right]\right)$. This operation can be iterated with more set splitting operators $\sigma_{3}, \ldots$, and leads to the multi-stage segmentation strategy introduced by Serra $[22,17]$. This construction will be analysed in detail in Subsection 3.2.

Finally, consider the extraction of large objects that can have small protruding parts. In Figure 2, the two sets $A$ and $B$ are each one large object with four protrusions, so each one will be extracted as a whole, in other words, $\sigma(A)=\mathbf{1}_{A}$ and $\sigma(B)=\mathbf{1}_{B}$; now since $A$ and $B$ overlap, for $\sigma$ isotone we would 


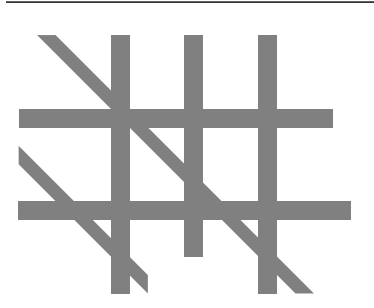

(a)

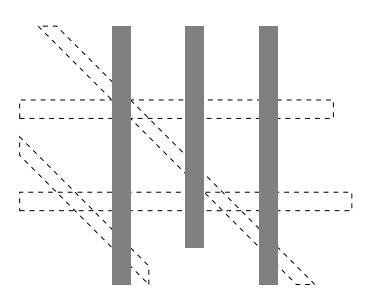

(d)



(b)

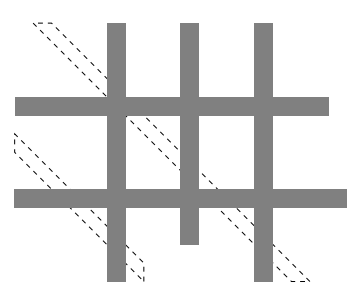

(e)



(c)

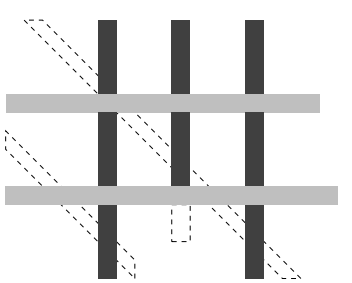

(f)

Fig. 1 Here $E=\mathbf{Z}^{2}$. (a) A set $A$ from which horizontal and vertical bars are to be extracted. (b) The two structuring elements $H$ and $V$. (c) The partial partition $\operatorname{PC}^{\mathcal{C}_{H}^{*}}(A)$ of $A$ into its $\mathcal{C}_{H}^{*}$-components of $A$ (connected components of the opening $A \circ H$ ), gives the 2 horizontal bars. (d) The partial partition $\mathrm{PC}^{\mathcal{C}_{V}^{*}}(A)$ of $A$ into its $\mathcal{C}_{V}^{*}$-components (connected components of $A \circ V)$, gives the 3 vertical bars. (e) Their join $\mathrm{PC}^{\mathcal{C}_{H}^{*}}(A) \vee \mathrm{PC}^{\mathcal{C}_{V}^{*}}(A)$; since $\mathrm{PC}^{\mathcal{C}_{H}^{*}}(A)$ and $\mathrm{PC}^{\mathcal{C}_{V}^{*}}(A)$ are invariant under any opening extracting horizontal and vertical objects, so must be $\mathrm{PC}^{\mathcal{C}_{H}^{*}}(A) \vee \mathrm{PC}^{\mathcal{C}_{V}^{*}}(A)$, but then the horizontal and vertical bars are not separated. (f) In order to extract separately the horizontal and vertical bars, we first take $\mathrm{PC}^{\mathcal{C}_{H}^{*}}(A)$, giving the 2 horizontal bars (in light grey), then apply $\mathrm{PC}^{\mathcal{C}_{V}^{*}}$ to the residual $A \backslash \operatorname{supp}\left[\mathrm{PC} \mathcal{C}_{H}^{*}(A)\right]=$ $A \backslash(A \circ H)$, giving 8 remaining vertical bars (in dark grey).

have $\sigma(A \cup B)=\mathbf{1}_{A} \vee \mathbf{1}_{B}=\mathbf{1}_{A \cup B}$, while in fact $\sigma(A \cup B)$ should contain two objects separated by a narrow transition.



(a)

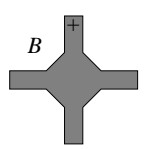

(b)

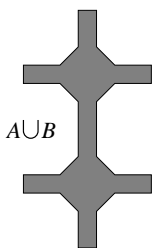

(c)

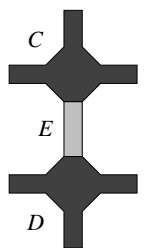

(d)

Fig. 2 (a) and (b) The two sets $A$ and $B$, where the + indicates the position of the origin. Since $A$ and $B$ represent each one object, we have $\sigma(A)=\mathbf{1}_{A}$ and $\sigma(B)=\mathbf{1}_{B}$. (c) If $\sigma$ was isotone, we would have $\sigma(A \cup B)=\mathbf{1}_{A \cup B}$. (d) However $A \cup B$ consists of two objects $C$ and $D$ (in dark grey) separated by a transition $E$ (in light grey), thus $\sigma(A \cup B)=\mathbf{1}_{C} \vee \mathbf{1}_{D}$.

A similar problem is the so-called "trend effect" in the segmentation of grey-level images [28]: an image where the intensity varies slowly from dark to bright over a wide area will globally look heterogeneous, while locally every 
small region will look homogeneous. In both cases a possible solution is to use Soille's segmentation approach [27-30] studied in Subsection 3.3: (a) build a growing sequence of segmentations $\pi_{1} \leq \cdots \leq \pi_{n}$ by using growing sequence of partial connections (e.g., arising from growing criteria); (b) in each partial partition $\pi_{i}(i=1, \ldots, n)$, remove all blocks that do not satisfy some constraint, leading to a filtered partial partition $\pi_{i}^{\prime}$; (c) take as final segmentation the join $\bigvee_{i=1}^{n} \pi_{i}^{\prime}$. See Figure 10 for the segmentation of a function, and also Figures 11 and 12 for the decomposition of a set into two wide parts joined by a narrow transition.

This second paper will thus investigate non-isotone idempotent block splitting operators on partial partitions. Idempotence is required for the stability of the segmentation process (a non-homogeneous set is split into homogeneous subsets that are not split further). In fact, all idempotent operators that we construct belong to two families of operators that we introduced before under the names of open-condensations [11] and open-overcondensations [12]. For the sake of brevity, we rename them here $C$-thinnings and $O C$-thinnings respectively. They are order-theoretical generalizations of openings, and have several similarities with them, notably they constitute Moore families.

We give a few methods for constructing such idempotent block splitting operators from elementary operations on blocks. They are based on the opening $\mathrm{CS}^{\mathcal{C}}$ decomposing each block into its $\mathcal{C}$-components (for a partial connection $\mathcal{C})$. First, $\mathrm{CS}^{\mathcal{C}}$ can either be preceded by a feature extraction operator $[2,8$, 9], see Proposition 8 , or it can be followed by a block selection operator as in Soille's approach to segmentation [28], see Subsection 3.3; both operations produce OC-thinnings. Next, we propose an associative binary operation on set splitting operators, the residual combination, where the second operator is applied to the "residue" of the first one; this operation was introduced in [22] in the specific case of the decomposition into $\mathcal{C}$-components, and we show that the resulting operation on block splitting operators preserves the family of C-thinnings, see Subsection 3.2. Finally, the lattice-theoretical join preserves both families of C-thinnings and OC-thinnings; this is in particular relevant to Soille's approach [28].

In Section 4, we will indicate the relevance for image segmentation (or shape decomposition) of various order-theoretical properties of block splitting operators.

\subsection{Paper organization}

We adopt the general terminology (inherited from [15]) of the first paper, which follows that of mathematical morphology $[19,5,26]$, except when it contradicts classical lattice-theoretical usage.

Section 2 gives the mathematical background: we first recall the main notation (Table 1), results and formulas from the first paper that we will use here; Table 2 lists the notation introduced in this paper (in the order of first appearance); then we describe $C$-thinnings (open-condensations), $O C$-thinnings 
(open-overcondensations) and trivial operators, and give their main properties. Section 3 considers idempotent non-isotone block splitting operators, in particular the residual combination of block splitting openings introduced by [22] and the operators underlying Soille's approach to segmentation [28]. Finally Section 4 discusses the significance of our results, concludes and proposes new tracks for research.

Although the operators described in Section 3 are based on existing image segmentation algorithms, all properties proved about them are new.

\section{Background}

Table 1 summarizes the main notation introduced in the first paper. We now briefly recall the main results and formulas from it that will be needed in this second work. Then Subsection 2.1 will describe some families of latticetheoretical operators, in particular those introduced by the author $[11,12]$.

We first give a small simplification of notation: for an operator $\psi$ on $\mathcal{P}(E)$ and a point $p \in E$, write $\psi(p)$ for $\psi(\{p\})$.

Recall now the basic morphological operators on subsets of $E=\mathbf{R}^{m}$ or $E=\mathbf{Z}^{m}$; for any $B \in \mathcal{P}(E)$, we have the following four operators on $\mathcal{P}(E)$ : the dilation by $B, \delta_{B}: X \mapsto X \oplus B$; the erosion by $B, \varepsilon_{B}: X \mapsto X \ominus B$; the opening by $B, \gamma_{B}=\delta_{B} \varepsilon_{B}: X \mapsto X \circ B=(X \ominus B) \oplus B$; the closing by $B$, $\varphi_{B}=\varepsilon_{B} \delta_{B}: X \mapsto X \bullet B=(X \oplus B) \ominus B$. We write $B_{p}$ for the translate of $B$ by a point $p \in E$; we have $B_{p}=\delta_{B}(p)$.

Given a a non-empty family $\pi_{i} \in \Pi^{*}(E), i \in I \neq \emptyset$, such that for every $p \in E$, the set of $\mathrm{Cl}_{\pi_{i}}(p)$ for $i \in I$ is directed (i.e., $\forall i, j \in I, \exists k \in I, \mathrm{Cl}_{\pi_{i}}(p) \cup$ $\left.\mathrm{Cl}_{\pi_{j}}(p) \subseteq \mathrm{Cl}_{\pi_{k}}(p)\right)$, we have [15]

$$
\forall p \in E, \quad \mathrm{Cl}_{i \in I} \pi_{i}(p)=\bigcup_{i \in I} \mathrm{Cl}_{\pi_{i}}(p)
$$

and for any $\pi \in \Pi^{*}(E), \pi \wedge\left(\bigvee_{i \in I} \pi_{i}\right)=\bigvee_{i \in I}\left(\pi \wedge \pi_{i}\right)$. An elementary consequence of this is:

$$
\forall \pi, \pi^{\prime} \in \Pi^{*}(E), \quad \pi \wedge \pi^{\prime}=\bigvee_{B \in \pi}\left(\mathbf{1}_{B} \wedge \pi^{\prime}\right)
$$

Given a fixed $\pi^{*} \in \Pi^{*}(E)$ and $B \in \pi^{*}$, for any $\pi \in \Pi^{*}(E)$ such that $\pi \leq \pi^{*}$, the restriction of $\pi$ to $B$ is the set $[\pi]_{B}$ of blocks of $\pi$ that are included in $B$; we have

$$
\forall \pi \leq \pi^{*}: \quad \pi=\bigcup_{B \in \pi^{*}}[\pi]_{B}=\bigvee_{B \in \pi^{*}}[\pi]_{B}
$$

This restriction is compatible with refinement order and with the supremum and infimum operations. 
Table 1 Main notation from the first paper

\begin{tabular}{|c|c|}
\hline$E$ & space of points \\
\hline$T$ & set of image values (grey-levels or colours) \\
\hline $\mathrm{cr}$ & a criterion $T^{E} \times \mathcal{P}(E) \rightarrow\{0,1\}$ \\
\hline $\mathcal{C}_{\mathrm{cr}}^{F}$ & $\{A \in \mathcal{P}(E) \mid \operatorname{cr}[F, A]=1\}\left(F \in T^{E}\right)$ \\
\hline$(\alpha, \beta): A \rightleftharpoons B$ & $\alpha: A \rightarrow B$ and $\beta: B \rightarrow A$ \\
\hline $\operatorname{lnv}(\psi)$ & invariance domain of the operator $\psi$ \\
\hline $\operatorname{supp}(\mathcal{B})$ & support of the family $\mathcal{B}$ of subsets of $E$ \\
\hline$\pi$ & a partial partition \\
\hline $\mathrm{Cl}_{\pi}(p)$ & class of point $p$ in the partial partition $\pi$ \\
\hline$\Pi(E)$ & set of all partitions of $E$ \\
\hline$\Pi^{*}(E)$ & set of all partial partitions of $E$ \\
\hline$\varnothing$ & empty partial partition \\
\hline $\mathbf{0}_{A}$ & identity partition of $A$ into its singletons \\
\hline $\mathbf{1}_{A}$ & universal partition of $A$ into a single block \\
\hline 1. & $A \mapsto \mathbf{1}_{A}$ \\
\hline 0 . & $A \mapsto \mathbf{0}_{A}$ \\
\hline $\mathcal{S}(E)$ & family of all singletons in $E$ \\
\hline $\mathcal{C}$ & a partial connection on $\mathcal{P}(E)$ \\
\hline$\gamma_{p}$ & partial connection opening on $\mathcal{P}(E)$ \\
\hline$\left(\gamma_{p}, p \in E\right)$ & system of partial connection openings on $\mathcal{P}(E)$ \\
\hline $\mathrm{PC}^{\mathcal{C}}(X)$ & partial partition of all $\mathcal{C}$-components of $X$ \\
\hline $\mathcal{C}_{\text {std }}$ & $\begin{array}{l}\text { a "standard" connection on } \mathcal{P}(E) \\
\text { (e.g., arc, topological, or graph connectivity) }\end{array}$ \\
\hline $\begin{array}{l}\mathcal{C}_{B}^{*} \\
A E(L)\end{array}$ & $\begin{array}{l}\text { partial connection }\left\{Z \in \mathcal{C}_{\text {std }} \mid Z \circ B=Z\right\}\left(B \in \mathcal{C}_{\text {std }}, B \neq \emptyset\right) \\
\text { set of all anti-extensive operators on } L\end{array}$ \\
\hline$[\pi]_{B}$ & $\pi \cap \mathcal{P}(B)$, for $B \in \pi^{*}, \pi \leq \pi^{*}$ \\
\hline$\sigma$ & set splitting operator $X \mapsto \sigma(X) \in \Pi^{*}(X)$ \\
\hline$\beta(\sigma)$ & block splitting operator on $\Pi^{*}(E)$ derived from $\sigma$ \\
\hline $\mathbf{1}_{\psi}$ & set shrinking operator $X \mapsto \mathbf{1}_{\psi(X)}$ \\
\hline $\mathrm{B}(\psi)$ & $\pi \mapsto \bigvee_{B \in \pi} \mathbf{1}_{\psi(B)}$ \\
\hline$\Pi^{*}(E, \mathcal{C})$ & set of all partial partitions of $E$ with blocks in $\mathcal{C} \backslash\{\emptyset\}$ \\
\hline $\mathrm{CS}^{\mathrm{C}}$ & opening on $\Pi^{*}(E)$ splitting blocks into $\mathcal{C}$-components \\
\hline $\mathcal{F}(\sigma)$ & $\left\{X \in \mathcal{P}(E) \mid \sigma(X)=\mathbf{1}_{X}\right\}$, fixed set of $\sigma$ \\
\hline
\end{tabular}

Table 2 Notation introduced in this paper

\begin{tabular}{ll}
\hline $\mathrm{p}$ & a predicate $L \backslash\{\mathbf{0}\} \rightarrow\{0,1\}$ \\
$\tau_{\mathrm{p}}$ & trivial operator corresponding to $\mathrm{p}$ \\
$\mathrm{rc}[\sigma, \theta]$ & residual combination of $\sigma$ followed by $\theta$ \\
$\mathrm{rc}\left[\sigma_{1}, \ldots, \sigma_{n}\right]$ & residual combination of the succession of $\sigma_{1}, \ldots, \sigma_{n}$ \\
$\pi^{1}-\pi^{2}$ & $\left\{B \backslash \operatorname{supp}\left(\left[\pi^{2}\right]_{B}\right) \mid B \in \pi^{1}, B \backslash \operatorname{supp}\left(\left[\pi^{2}\right]_{B}\right) \neq \emptyset\right\}$, for $\pi^{1} \geq \pi^{2}$ \\
$\pi^{1}+\pi^{2}$ & $\pi^{1} \cup \pi^{2}$, for $\operatorname{supp}\left(\pi^{1}\right) \cap \operatorname{supp}\left(\pi^{2}\right)=\emptyset$
\end{tabular}

Given a set splitting operator $\sigma: \mathcal{P}(E) \rightarrow \Pi^{*}(E): X \mapsto \sigma(X) \in \Pi^{*}(X)$, the block splitting operator derived from $\sigma$ is $\beta(\sigma): \Pi^{*}(E) \rightarrow \Pi^{*}(E)$ given by

$$
\forall \pi \in \Pi^{*}(E), \quad \beta(\sigma)(\pi)=\bigcup_{B \in \pi} \sigma(B)=\bigvee_{B \in \pi} \sigma(B)
$$


then for all $B \in \pi$ we have $[\beta(\sigma)(\pi)]_{B}=\sigma(B)$. For any $\pi \in \Pi^{*}(E)$ and $p \in E$, we have $\mathrm{Cl}_{\beta(\sigma)(\pi)}(p)=\mathrm{Cl}_{\sigma\left(\mathrm{Cl}_{\pi}(p)\right)}(p)$. Note that for any $A \in \mathcal{P}(E)$, $\beta(\sigma)\left(\mathbf{1}_{A}\right)=\sigma(A)$.

Given an anti-extensive operator $\psi$ on $\mathcal{P}(E)$, the set shrinking operator $\mathbf{1}_{\psi}: X \mapsto \mathbf{1}_{\psi(X)}$ is a special case of set splitting operator; it gives rise to the block shrinking operator $\beta\left(\mathbf{1}_{\psi}\right)$, which coincides with $\mathrm{B}(\psi)$, the blockwise extension of $\psi$. For any $\pi \in \Pi^{*}(E)$,

$$
\mathrm{B}(\psi)(\pi)=\bigvee_{B \in \pi} \mathbf{1}_{\psi(B)}=\{\psi(B) \mid B \in \pi, \psi(B) \neq \emptyset\}
$$

For any $\pi \in \Pi^{*}(E)$ and $p \in E$, we have

$$
\mathrm{Cl}_{\mathrm{B}(\psi)(\pi)}(p)= \begin{cases}\psi\left(\mathrm{Cl}_{\pi}(p)\right) & \text { if } p \in \psi\left(\mathrm{Cl}_{\pi}(p)\right) \\ \emptyset & \text { otherwise }\end{cases}
$$

Note that a supremum, infimum or composition of block splitting operators is block splitting. The same can be said for an infimum or composition of block shrinking operators.

For any set splitting operator $\sigma$ on $\mathcal{P}(E)$, the invariance domain of $\beta(\sigma)$, that is, the set of $\pi \in \Pi^{*}(E)$ such that $\beta(\sigma)(\pi)=\pi$, is

$$
\operatorname{lnv}(\beta(\sigma))=\Pi^{*}(E, \mathcal{F}(\sigma)), \text { where } \mathcal{F}(\sigma)=\left\{X \in \mathcal{P}(E) \mid \sigma(X)=\mathbf{1}_{X}\right\}
$$

and we have $\emptyset \in \mathcal{F}(\sigma)$.

A typical set splitting operator is, given a partial connection $\mathcal{C}$, the map $\mathrm{PC}^{\mathcal{C}}$ associating to a set $A$ the partial partition $\mathrm{PC}^{\mathcal{C}}(A)$ of its $\mathcal{C}$-components. Then $\beta\left(\mathrm{PC}^{\mathcal{C}}\right)=\mathrm{CS}^{\mathcal{C}}$, the operator on $\Pi^{*}(E)$ that splits each block of a partial partition into its $\mathcal{C}$-components:

$$
\begin{aligned}
\forall \pi \in \Pi^{*}(E), \quad \operatorname{CS}^{\mathcal{C}}(\pi) & =\bigcup_{C \in \pi} \operatorname{PC}^{\mathcal{C}}(C) \\
& =\left\{\gamma_{p}(C) \mid C \in \pi, p \in C, \gamma_{p}(C) \neq \emptyset\right\} .
\end{aligned}
$$

Given $\left(\gamma_{p}, p \in E\right)$ the system of partial connection openings of $\mathcal{C}$, we have:

$$
\forall \pi \in \Pi^{*}(E), \forall p \in E, \quad \mathrm{Cl}_{\mathrm{CS}^{\mathcal{C}}(\pi)}(p)=\mathrm{Cl}_{\mathrm{PC}^{\mathcal{C}}\left(\mathrm{Cl}_{\pi}(p)\right)}(p)=\gamma_{p}\left(\mathrm{Cl}_{\pi}(p)\right)
$$

Now $\mathrm{CS}^{\mathcal{C}}$ is an opening on $\Pi^{*}(E)$, whose invariance domain is $\Pi^{*}(E, \mathcal{C})$.

For $E=\mathbf{R}^{m}$ or $\mathbf{Z}^{m}$, given a "standard" connection $\mathcal{C}_{\text {std }}$ on $\mathcal{P}(E)$, taking a non-void $B \in \mathcal{C}_{s t d}$, the set $\mathcal{C}_{B}^{*}$ of all $Z \in \mathcal{C}_{s t d}$ such that $Z \circ B=Z$ is a partial connection; for $X \notin \mathcal{C}_{B}^{*}$, the $\mathcal{C}_{B}^{*}$-components of $X$ are the $\mathcal{C}_{\text {std }}$-components of the opening $X \circ B$, see Figure 6 (b). 
2.1 Some special types of operators

We analyse here some families of operators having specialized properties: first $C$-thinnings (open-condensations [11]) and OC-thinnings (open-overcondensations [12]), then trivial operators. They have been considered in older papers, but we will find them again throughout Section 3, when studying some block splitting operators on partial partitions that are relevant to image segmentation.

We will see that C-thinnings and OC-thinnings are in some way two generalizations of openings, sharing some features with them.

Definition 1 Let $L$ and $M$ be two posets (equal or distinct).

1. An operator $\eta: L \rightarrow M$ is

- condensing [11] if for any $x, y, z \in L$ such that $x \leq y \leq z$ and $\eta(x)=$ $\eta(z)$, we have $\eta(x)=\eta(y)$;

- overcondensing [12] if for any $x, y, z \in L$ such that $x \leq y \leq z$ and $\eta(x)=\eta(z)$, we have $\eta(x) \leq \eta(y)$.

2. An operator on $L$ is

- a thinning if it is anti-extensive and idempotent (J. Serra);

- a C-thinning (in [11]: an open-condensation) if it is condensing, antiextensive and idempotent;

- an OC-thinning (in [12]: an open-overcondensation) if it is overcondensing, anti-extensive and idempotent.

An isotone operator is condensing, and a condensing operator is overcondensing; every opening is a C-thinning, and every C-thinning is an OCthinning. We have the following practical characterization:

Lemma 2 [11,12] Let $\kappa$ be an operator on the poset L. Then:

$-\kappa$ is a C-thinning if and only if it is anti-extensive and satisfies the following condition:

$(\diamond)$ For $x, y \in L, \kappa(x) \leq y \leq x \Rightarrow \kappa(x)=\kappa(y)$.

$-\kappa$ is an OC-thinning if and only if it is anti-extensive and satisfies the following condition:

( For $x, y \in L, \kappa(x) \leq y \leq x \Rightarrow \kappa(x) \leq \kappa(y)$.

Let us now extend to C-thinnings and OC-thinnings two methods that have been used for constructing openings:

Proposition 3 Let $L$ and $M$ be two posets (equal or distinct). Then consider the following two constructions of an operator $\kappa^{\prime}$ on $L$ :

1. Given an opening $\gamma$ on $L$ and an operator $\kappa$ on $L$, such that $\gamma \kappa \gamma=\kappa \gamma$, let $\kappa^{\prime}=\kappa \gamma$.

2. Given an adjunction $(\varepsilon, \delta): L \rightleftharpoons M$ and an operator $\kappa$ on $M$, let $\kappa^{\prime}=\delta \kappa \varepsilon$.

In both cases, if $\kappa$ is a C-thinning (resp., an OC-thinning), then $\kappa^{\prime}$ is a $C$ thinning (resp., an OC-thinning). 
Proof We show the result for an OC-thinning. For a C-thinning, the proof is the same, replacing $(\star)$ by $(\diamond)$.

1. As $\gamma$ and $\kappa$ are anti-extensive, for $x \in L$ we have $\kappa \gamma(x) \leq \gamma(x) \leq x$, thus $\kappa \gamma$ is anti-extensive. Let $x, y \in L$ such that $\kappa \gamma(x) \leq y \leq x$. As $\gamma$ is isotone, $\gamma \kappa \gamma(x) \leq \gamma(y) \leq \gamma(x)$, and as $\gamma \kappa \gamma=\kappa \gamma, \kappa \gamma(x) \leq \gamma(y) \leq \gamma(x)$. Applying $(\star)$ with $\gamma(x)$ and $\gamma(y)$ instead of $x$ and $y$, we get $\kappa \gamma(y) \geq \kappa \gamma(x)$. Hence $\kappa \gamma$ satisfies $(\star)$, so it is an OC-thinning.

2. As $\kappa$ is anti-extensive, for $x \in L$ we have $\kappa \varepsilon(x) \leq \varepsilon(x)$; then the adjunction $(\varepsilon, \delta)$ gives $\delta \kappa \varepsilon(x) \leq x$, thus $\delta \kappa \varepsilon$ is anti-extensive. Let $x, y \in L$ such that $\delta \kappa \varepsilon(x) \leq y \leq x$. By the adjunction $(\varepsilon, \delta), \kappa \varepsilon(x) \leq \varepsilon(y)$, and as $\varepsilon$ is isotone, $\varepsilon(y) \leq \varepsilon(x)$. So $\kappa \varepsilon(x) \leq \varepsilon(y) \leq \varepsilon(x)$, and applying $(\star)$ with $\varepsilon(x)$ and $\varepsilon(y)$ instead of $x$ and $y$, we get $\kappa \varepsilon(y) \geq \kappa \varepsilon(x)$. As $\delta$ is isotone, $\delta \kappa \varepsilon(y) \geq \delta \kappa \varepsilon(x)$. Hence $\delta \kappa \varepsilon$ satisfies $(\star)$, so it is an OC-thinning.

It is indeed well-known that for an opening $\kappa$, both constructions for $\kappa^{\prime}$ give an opening. The analogy with openings is reinforced by the fact that a supremum of C-thinnings is a C-thinning, and similarly for OC-thinnings; in other words, in a complete lattice, $C$-thinnings and $O C$-thinnings both constitute dual Moore families $[11,12]$.

A classical example of OC-thinning on sets is the so-called generalized foreground opening introduced by [2]. Let $E=\mathbf{R}^{m}$ or $E=\mathbf{Z}^{m}$, given two disjoint $A, B \in \mathcal{P}(E)$, the hit-or-miss transform by $(A, B)$ is the map

$$
X \mapsto(X \ominus A) \cap\left(X^{c} \ominus B\right)=\left\{p \in E \mid A_{p} \in X, B_{p} \in X^{c}\right\} ;
$$

here $A$ and $B$ are respectively the foreground and background structuring elements. Now consider the following operator on $\mathcal{P}(E)$ :

$$
X \mapsto\left[(X \ominus A) \cap\left(X^{c} \ominus B\right)\right] \oplus A=\bigcup\left\{A_{p} \mid p \in E, A_{p} \in X, B_{p} \in X^{c}\right\}
$$

namely the composition of the hit-or-miss transform by $(A, B)$ followed by the dilation by $A$; it is an OC-thinning [12], and it is used to extract shapes from a binary image. It has been called generalized foreground opening [2] or hit-or-miss opening [26], by analogy with the opening by $A, X \mapsto(X \ominus A) \oplus A$.

As a generalization, assuming $M$ to be a meet semilattice: given an adjunction $(\varepsilon, \delta): L \rightleftharpoons M$ and an antitone operator $\theta: L \rightarrow M, \delta(\varepsilon \wedge \theta)$ is an OC-thinning on $L$; furthermore, if $L$ is a complete lattice, then every OCthinning on $L$ takes this form for some complete lattice $M$ [12]. Generalizing further, we deduce that given an adjunction $(\varepsilon, \delta): L \rightleftharpoons M$, an opening $\gamma$ on $M$ and an antitone operator $\theta: L \rightarrow M, \delta \gamma(\varepsilon \wedge \theta)$ is an OC-thinning on $L$.

All this justifies thus the idea proposed in [12] that OC-thinnings can be considered as a kind of non-isotone generalization of openings.

Quasi-openings can be built with the techniques of [11]. The first one is the toggle of openings, for example in $\mathcal{P}(E)$, given several openings $\gamma_{i}(i \in I)$, for every $X \in \mathcal{P}(E)$, choose the $\gamma_{i}(X)$ having the largest size. The second originates from [10], we called it the Pitas decomposition: given two openings $\gamma$ and $\gamma^{\prime}$ on $\mathcal{P}(E)$, take the operator $X \mapsto \gamma(X) \cup \gamma^{\prime}(X \backslash \gamma(X))$. This construction 
was generalized in [11] as follows: let $(G,+, \leq)$ be an abelian partially ordered group with neutral element 0 , and let $P$ be the poset of all positive elements of $G(x \in G$ such that $x \geq 0)$, then given two C-thinnings $\gamma, \gamma^{\prime}$ on $P$, the map $\kappa: x \mapsto \gamma(x)+\gamma^{\prime}(x-\gamma(x))$ is defined on $P$, and is a C-thinning. In Subsection 3.2, we will define a similar construction for partial partitions, so we need a further generalization:

Proposition 4 Let $P$ be a poset and let + and - be two partially defined binary operations on $P$ such that for $w, x, y, z \in P$ we have:

for $y \leq x$, (a) $x-y$ is defined, and (b) if $y \leq x \leq w$, then $x-y \leq w-y$; for $y \leq x$ and $z \leq x-y$, (c) $y+z$ is defined, (d) $y+z \leq x$, (e) $y \leq y+z$ and $(f)(y+z)-y=z$.

Given two anti-extensive operators $\eta, \zeta$ on $P$, for every $x \in P$ the expression $\eta(x)+\zeta(x-\eta(x))$ is defined, and the operator $\kappa: x \mapsto \eta(x)+\zeta(x-\eta(x))$ on $P$ is anti-extensive. Furthermore, if $\eta$ and $\zeta$ are $C$-thinnings, then $\kappa$ is a C-thinning; more precisely, for $\kappa(x) \leq y \leq x$, we have $\eta(y)=\eta(x)$ and $\zeta(y-\eta(y))=\zeta(x-\eta(x))$.

Proof Let $x \in P$. Since $\eta$ is anti-extensive, we have $\eta(x) \leq x$, so $x-\eta(x)$ is defined by (a). As $\zeta$ is anti-extensive, $\zeta(x-\eta(x)) \leq x-\eta(x)$, so $\kappa(x)=$ $\eta(x)+\zeta(x-\eta(x))$ is defined by (c) with $y=\eta(x)$ and $z=\zeta(x-\eta(x))$. Thus $\kappa$ is well-defined. Then by $(\mathrm{d})$ we have $\kappa(x) \leq x$, so $\kappa$ is anti-extensive.

Now (e) gives $\eta(x) \leq \kappa(x)$, and by (f) we get $\kappa(x)-\eta(x)=\zeta(x-\eta(x))$. Suppose that $\eta$ and $\zeta$ are C-thinnings, and let $y \in P$ such that that $\kappa(x) \leq$ $y \leq x$. As $\eta(x) \leq \kappa(x)$, we have $\eta(x) \leq y \leq x$, and since $\eta$ is a C-thinning, we obtain $\eta(y)=\eta(x)$. Since $\eta(x) \leq \kappa(x) \leq y \leq x$, by (b) we have $\kappa(x)-\eta(x) \leq$ $y-\eta(x) \leq x-\eta(x) ;$ but $\kappa(x)-\eta(x)=\zeta(x-\eta(x))$, so $\zeta(x-\eta(x)) \leq y-\eta(x) \leq$ $x-\eta(x)$. As $\zeta$ is a C-thinning, we obtain $\zeta(y-\eta(x))=\zeta(x-\eta(x))$, and since $\eta(y)=\eta(x)$, this gives $\zeta(y-\eta(y))=\zeta(x-\eta(x))$. Hence $\kappa(y)=\eta(y)+\zeta(y-$ $\eta(y))=\eta(x)+\zeta(x-\eta(x))=\kappa(x)$. We have thus shown that $\kappa$ satisfies $(\diamond)$, so it is a C-thinning.

The first case where this result applies is the situation of [11], where $P$ is the set of positive $(\geq 0)$ elements in an abelian p.o. group $(G,+, \leq)$, with + and - denoting the addition and subtraction operations; since + and - are always defined in $G$, here " $x-y$ is defined" should be interpreted as " $x-y \in P$ ", i.e., $x-y \geq 0$, while for positive $y$, $z$, we always have $y+z \geq 0$, i.e., $y+z \in P$; then conditions $(\mathrm{a}-\mathrm{f})$ are straightforward consequences of the compatibility of the addition with the order $\leq$, and of the fact that we take positive elements. An example is when $G$ is the set of functions $E \rightarrow \mathbf{R}$ (where $\mathbf{R}$ is the set of real numbers) and $P$ is the subset of functions $E \rightarrow \mathbf{R}^{+}$(where $\mathbf{R}^{+}$is the set of non-negative reals). Next, conditions $(\mathrm{a}-\mathrm{f})$ are also satisfied by the poset of functions $E \rightarrow\{0,1\}$, hence by the isomorphism $(\mathcal{P}(E), \subseteq) \leftrightarrow\left(2^{E}, \leq\right)$, we can apply the proposition to $\mathcal{P}(E)$, with $X+Y$ being the union of two disjoint sets $X$ and $Y$, and $X-Y$ being the subtraction of a subset $Y$ from a set $X$ : this gives the original Pitas decomposition of [10]. This case gives a new 
intuitive meaning for + and $-: x-y$ is the "residue" obtained by "removing" the part $y$ from the object $x$, and $x+y$ is the "join" of two non-overlapping objects $x$ and $y$. In Subsection 3.2 we will define + and - for partial partitions according to that meaning, cf. Proposition 11.

In mathematical morphology, one has considered $[4,6]$ operators that remove from a set all connected components that do not satisfy some property; in Subsection 3.3, we will discuss them in the framework of operators removing some blocks in a partial partition. Although they were usually considered in the lattice $\mathcal{P}(E)$, we can extend them to an arbitrary poset with least element:

Definition 5 Let $L$ be a poset with least element $\mathbf{0}$.


have $\mathrm{p}(x)=1$ if $x$ satisfies $\mathrm{p}$, and $\mathrm{p}(x)=0$ if $x$ does not satisfy $\mathrm{p}$.

2. A trivial operator on $L$ [25] is some $\tau: L \rightarrow L$ such that for any $x \in L$ we have $\tau(x)=x$ or $\tau(x)=\mathbf{0}$.

3. Given a predicate $\mathrm{p}$ on $L \backslash\{\mathbf{0}\}$, the trivial operator on $L$ corresponding to $\mathrm{p}$ is $\tau_{\mathrm{p}}$ given by:

$$
\begin{aligned}
& \forall x \in L \backslash\{\mathbf{0}\}, \tau_{\mathrm{p}}(x)=\left\{\begin{array}{ll}
x & \text { if } \mathrm{p}(x)=1 \\
\mathbf{0} & \text { if } \mathrm{p}(x)=0
\end{array} ;\right. \\
& \tau_{\mathrm{p}}(\mathbf{0})=\mathbf{0} .
\end{aligned}
$$

Note that a trivial operator gives always $\tau(\mathbf{0})=\mathbf{0}$; hence the predicate $\mathrm{p}$ needs not to be defined on $\mathbf{0}$, because $\tau_{\mathrm{p}}(\mathbf{0})=\mathbf{0}$ whatever the value of $\mathbf{p}(\mathbf{0})$.

Proposition 6 Let $L$ be a poset with least element $\mathbf{0}$. The map $\mathrm{p} \mapsto \tau_{\mathrm{p}}$ given in (11) is a bijection between predicates on $L \backslash\{\mathbf{0}\}$ and trivial operators on $L$, the inverse bijection is $\tau \mapsto \mathrm{p}_{\tau}$, where

$$
\forall x \in L \backslash\{\mathbf{0}\}, \quad \mathbf{p}_{\tau}(x)=\left\{\begin{array}{ll}
1 & \text { if } \tau(x)=x \\
0 & \text { if } \tau(x)=\mathbf{0}
\end{array} .\right.
$$

This map $\mathrm{p} \mapsto \tau_{\mathrm{p}}$ is an order-embedding, i.e., $\mathrm{p} \leq \mathrm{q} \Leftrightarrow \tau_{\mathrm{p}} \leq \tau_{\mathrm{q}}$; when $L$ is a complete lattice, this map is a complete embedding (i.e., an injective complete morphism) from the complete lattice of predicates to $A E(L)$, the one of anti-extensive operators on $L$.

Furthermore, for any predicate $\mathrm{p}$ on $L \backslash\{\mathbf{0}\}$ :

1. $\tau_{\mathrm{p}}$ is an OC-thinning.

2. [25] $\tau_{\mathrm{p}}$ is an opening iff it is isotone, i.e., iff $\mathrm{p}$ is isotone on $L \backslash\{\mathbf{0}\}$.

3. $\tau_{\mathrm{p}}$ is a C-thinning iff it is an opening.

Proof Indeed, we check that $\mathrm{p}_{\tau_{\mathrm{p}}}=\mathrm{p}$ and $\tau_{\mathrm{p}_{\tau}}=\tau$, hence we have a bijection and its inverse. Clearly $\mathrm{p} \leq \mathrm{q} \Leftrightarrow \tau_{\mathrm{p}} \leq \tau_{\mathrm{q}}$. When $L$ is a complete lattice, we check that for a non-void family $\mathrm{p}_{i}(i \in I \neq \emptyset)$, we have

$$
\tau \bigvee_{i \in I} \mathrm{p}_{i}=\bigvee_{i \in I} \tau_{\mathrm{p}_{i}} \text { and } \tau \bigwedge_{i \in I} \mathrm{p}_{i}=\bigwedge_{i \in I} \tau_{\mathrm{p}_{i}}
$$


Finally the least and greatest predicates, which are constant 0 and constant 1, lead to the following trivial operators: the constant $\mathbf{0}$ operator and the identity; these are indeed the least and greatest anti-extensive operators.

We consider now the three other properties:

1. Clearly a trivial operator is anti-extensive. Let $x, y \in L$ such that $\tau_{\mathrm{p}}(x) \leq y \leq x$. If $\tau_{\mathrm{p}}(x)=x$, then $y=x$, so $\tau_{\mathrm{p}}(y)=\tau_{\mathrm{p}}(x)=x=y$. If $\tau_{\mathrm{p}}(x)=\mathbf{0}$, then $\tau_{\mathrm{p}}(y) \geq \mathbf{0}=\tau_{\mathrm{p}}(x)$. Thus the condition $(\star)$ of Lemma 2 is satisfied, and $\tau_{\mathrm{p}}$ is an OC-thinning.

2. The first equivalence follows from item 1 , the second one is well-known [25].

3. If $\tau_{\mathrm{p}}$ is an opening, then it is a C-thinning. Suppose now that $\tau_{\mathrm{p}}$ is a C-thinning. Let $x, y \in L \backslash\{\mathbf{0}\}$ such that $y \leq x$ and $\mathrm{p}(x)=0$; then $\tau_{\mathrm{p}}(x)=\mathbf{0}$, so $\tau_{\mathrm{p}}(x) \leq y \leq x$, and $(\diamond)$ gives $\tau_{\mathrm{p}}(y)=\tau_{\mathrm{p}}(x)=\mathbf{0}$, hence $\mathrm{p}(y)=0$; therefore $\mathrm{p}$ is isotone on $L \backslash\{\mathbf{0}\}$, so $\tau_{\mathrm{p}}$ is an opening by item 2 .

The trivial opening $\tau_{\mathrm{p}}$ corresponding to an isotone predicate $\mathrm{p}$ on $L \backslash\{\boldsymbol{0}\}$ has the invariance domain $\operatorname{lnv}\left(\tau_{\mathrm{p}}\right)=\{\mathbf{0}\} \cup \mathcal{U}$, where $\mathcal{U}=\{x \in L \backslash\{\mathbf{0}\} \mid \mathrm{p}(x)=1\}$ is an upper set. Conversely, every opening whose invariance domain is of the form $\{\mathbf{0}\} \cup \mathcal{U}$ for an upper set $\mathcal{U}$, is $\tau_{\mathrm{p}}$ for the isotone predicate $\mathrm{p}$ on $L \backslash\{\mathbf{0}\}$ defined by $\mathrm{p}(x)=1$ for $x \in \mathcal{U}$ and $\mathrm{p}(x)=0$ for $x \notin\{\mathbf{0}\} \cup \mathcal{U}$. Note that $\{\mathbf{0}\} \cup \mathcal{U}$ is a dual Moore family, hence a partial connection [13].

\section{Non-isotone block splitting}

In the first paper we have considered block splitting openings, namely the splitting of blocks into $\mathcal{C}$-components according to a partial connection $\mathcal{C}$. We will now study several types of non-isotone idempotent block splitting operators; they use block splitting openings as the main building block. Subsection 3.1 considers, in the case of block splitting operators, the properties of condensation and overcondensation; it gives also a construction for block splitting OC-thinnings. Subsection 3.2 analyses the residual combination of block splitting openings introduced by [22] and used in multi-stage segmentation approaches [17]; they are block splitting C-thinnings. Subsection 3.3 studies the combination of block splitting openings with trivial operators acting on blocks, following Soille's segmentation approach [27-30]; they are block splitting OC-thinnings. This leads also to a new interpretation of some operators on sets, namely the attribute thinnings or anti-extensive grain operators $[4,6]$.

\subsection{Overcondensation}

Let us first compare the properties of condensation and overcondensation for set and block splitting operators. Then we will construct block splitting OCthinnings by composing blockwise a union of generalized foreground openings (10) with the decomposition into $\mathcal{C}$-components. 
Proposition 7 Let $\sigma$ be a set splitting operator.

1. If $\beta(\sigma)$ is overcondensing, then $\sigma$ is overcondensing.

2. If $\beta(\sigma)$ is condensing, then $\sigma$ is condensing.

Let $\psi$ be an anti-extensive set operator.

3. $\mathrm{B}(\psi)$ is overcondensing iff $\psi$ is overcondensing.

4. If $\mathrm{B}(\psi)$ is condensing, then $\psi$ is condensing.

Proof 1. Suppose that $\beta(\sigma)$ is overcondensing. Let $X, Y, Z \in \mathcal{P}(E)$ such that $X \subseteq Y \subseteq Z$ and $\sigma(X)=\sigma(Z)$. Then $\mathbf{1}_{X} \leq \mathbf{1}_{Y} \leq \mathbf{1}_{Z}$, so $\beta(\sigma)\left(\mathbf{1}_{X}\right)=\sigma(X)=$ $\sigma(Z)=\beta(\sigma)\left(\mathbf{1}_{Z}\right)$. As $\beta(\sigma)$ is overcondensing, $\beta(\sigma)\left(\mathbf{1}_{X}\right) \leq \beta(\sigma)\left(\mathbf{1}_{Y}\right)$, that is, $\sigma(X) \leq \sigma(Y)$. Therefore $\sigma$ is overcondensing.

2. The proof is the same in the condensing case.

3. If $\mathrm{B}(\psi)=\beta\left(\mathbf{1}_{\psi}\right)$ is overcondensing, then $\mathbf{1}_{\psi}$ is overcondensing by item 1. Let $X, Y, Z \in \mathcal{P}(E)$ such that $X \subseteq Y \subseteq Z$ and $\psi(X)=\psi(Z)$; then $\mathbf{1}_{\psi(X)}=\mathbf{1}_{\psi(Z)}$, and as $\mathbf{1}_{\psi}$ is overcondensing, we get $\mathbf{1}_{\psi(X)} \leq \mathbf{1}_{\psi(Y)}$, hence $\psi(X) \subseteq \psi(Y)$. Therefore $\psi$ is overcondensing.

Suppose now that $\psi$ is overcondensing. Let $\pi^{0}, \pi^{1}, \pi^{2} \in \Pi^{*}(E)$ such that $\pi^{0} \leq \pi^{1} \leq \pi^{2}$ and $\mathrm{B}(\psi)\left(\pi^{0}\right)=\mathrm{B}(\psi)\left(\pi^{2}\right)$. Thus for any $p \in E, \mathrm{Cl}_{\pi^{0}}(p) \subseteq$ $\mathrm{Cl}_{\pi^{1}}(p) \subseteq \mathrm{Cl}_{\pi^{2}}(p)$ and $\mathrm{Cl}_{\mathrm{B}(\psi)\left(\pi^{0}\right)}(p)=\mathrm{Cl}_{\mathrm{B}(\psi)\left(\pi^{2}\right)}(p)$.

- If $\mathrm{Cl}_{\mathrm{B}(\psi)\left(\pi^{0}\right)}(p)=\emptyset$, then obviously $\mathrm{Cl}_{\mathrm{B}(\psi)\left(\pi^{0}\right)}(p) \subseteq \mathrm{Cl}_{\mathrm{B}(\psi)\left(\pi^{1}\right)}(p)$.

- If $\mathrm{Cl}_{\mathrm{B}(\psi)\left(\pi^{0}\right)}(p) \neq \emptyset$, then by $(6)$ we have $p \in \psi\left(\mathrm{Cl}_{\pi^{0}}(p)\right)$ and $\mathrm{Cl}_{\mathrm{B}(\psi)\left(\pi^{0}\right)}(p)=$ $\psi\left(\mathrm{Cl}_{\pi^{0}}(p)\right)$; we have also $p \in \psi\left(\mathrm{Cl}_{\pi^{2}}(p)\right)$ and $\mathrm{Cl}_{\mathrm{B}(\psi)\left(\pi^{2}\right)}(p)=\psi\left(\mathrm{Cl}_{\pi^{2}}(p)\right)$. Thus $\psi\left(\mathrm{Cl}_{\pi^{0}}(p)\right)=\psi\left(\mathrm{Cl}_{\pi^{2}}(p)\right)$, and as $\psi$ is overcondensing, we obtain $\psi\left(\mathrm{Cl}_{\pi^{0}}(p)\right) \subseteq \psi\left(\mathrm{Cl}_{\pi^{1}}(p)\right)$. We have then $p \in \psi\left(\mathrm{Cl}_{\pi^{1}}(p)\right)$, and by (6) again, $\mathrm{Cl}_{\mathrm{B}(\psi)\left(\pi^{1}\right)}(p)=\psi\left(\mathrm{Cl}_{\pi^{1}}(p)\right)$, hence $\mathrm{Cl}_{\mathrm{B}(\psi)\left(\pi^{0}\right)}(p) \subseteq \mathrm{Cl}_{\mathrm{B}(\psi)\left(\pi^{1}\right)}(p)$.

We have thus shown that in any case, $\mathrm{Cl}_{\mathrm{B}(\psi)\left(\pi^{0}\right)}(p) \subseteq \mathrm{Cl}_{\mathrm{B}(\psi)\left(\pi^{1}\right)}(p)$. As this holds for any $p \in E$, we get $\mathrm{B}(\psi)\left(\pi^{0}\right) \leq \mathrm{B}(\psi)\left(\pi^{1}\right)$. Therefore $\mathrm{B}(\psi)$ is overcondensing.

4. We use the same proof as in the first paragraph of item 3 .

Comparing items 3 and 4, the reciprocal of item 4 does not hold: we give an example of C-thinning $\psi$ such that $\mathrm{B}(\psi)$ is not condensing. Take two disjoint non-void subsets $A, B$ of $E$, where $B$ has size at least 2 . Define the operator $\psi$ on $\mathcal{P}(E)$ by

$$
\psi(X)=\left\{\begin{array}{ll}
A & \text { if } A \subseteq X, \\
B & \text { if } A \nsubseteq X \text { and } B \subseteq X \\
\emptyset & \text { if } A \nsubseteq X \text { and } B \nsubseteq X ;
\end{array} ;\right.
$$

then it is easily seen that $\psi$ is a C-thinning. Let $\{C, D\}$ be a partition of $B$ (i.e., $\emptyset \subset C \subset B$ and $D=B \backslash C)$. Then in $\Pi^{*}(E)$ we have $\{A, C, D\}<\{A, B\}<$ $\{A \cup B\}, \mathrm{B}(\psi)(\{A, C, D\})=\mathrm{B}(\psi)(\{A \cup B\})=\{A\}$, but $\mathrm{B}(\psi)(\{A, B\})=$ $\{A, B\}$, so $\mathrm{B}(\psi)$ is not a $\mathrm{C}$-thinning.

In fact, it can be shown (elaborating on the proof of item 3 ) that for a condensing anti-extensive operator $\psi$, the condition

$$
\forall X, Y \in \mathcal{P}(E), \quad Y \subseteq X \backslash \psi(X) \Longrightarrow \psi(Y)=\emptyset
$$


is sufficient for having $\mathrm{B}(\psi)$ condensing; when $\psi$ is idempotent (i.e., a Cthinning), this condition is also necessary.

Now comparing items 1 and 3, the reciprocal of item 1 does not hold: for a set splitting operator $\sigma$ that is not a set shrinking operator (i.e., is not of the form $\mathbf{1}_{\psi}$ for a set operator $\left.\psi\right), \sigma$ can be overcondensing while $\beta(\sigma)$ is not. Indeed, if $\sigma$ is support-preserving, for any $X \in \mathcal{P}(E)$ we have $\operatorname{supp}(\sigma(X))=X$, so $\sigma$ is injective, thus it is trivially condensing (hence overcondensing); but then $\beta(\sigma)$ is not necessarily overcondensing, as shows the example in Figure 3.
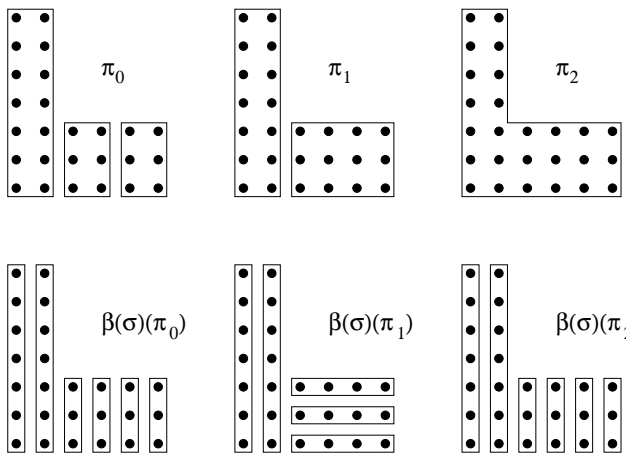



Fig. 3 Here $E=\mathbf{Z}^{2}$ and $\sigma$ splits a set $X \in \mathcal{P}(E)$ into its vertical crosssections if width $(X)<$ height $(X)<$ $\infty$, and into its horizontal crosssections otherwise. As $\sigma$ is supportpreserving, it is condensing. We have $\pi_{0} \leq \pi_{1} \leq \pi_{2}, \beta(\sigma)\left(\pi_{0}\right)=\beta(\sigma)\left(\pi_{2}\right)$, but $\beta(\sigma)\left(\pi_{0}\right) \not \subset \beta(\sigma)\left(\pi_{1}\right)$; hence $\beta(\sigma)$ is not overcondensing.

In any space $E$ provided with a partial connection $\mathcal{C}$, let $\left(\varepsilon_{i}, \delta_{i}\right)(i \in I)$ be a family of adjunctions on $\mathcal{P}(E)$ such that for every $i \in I$ and $p \in E$ we have $\delta_{i}(p) \in \mathcal{C}$. Now take the opening $\gamma=\bigvee_{i \in I} \delta_{i} \varepsilon_{i}$. For any $X \in \mathcal{P}(E)$, $\gamma(X)=\bigcup\left\{\delta_{i}(p) \mid i \in I, p \in E, \delta_{i}(p) \subseteq X\right\} ;$ since $\delta_{i}(p) \in \mathcal{C}, \delta_{i}(p) \subseteq X$ means that $\delta_{i}(p) \subseteq A$ for some $\mathcal{C}$-component $A$ of $X$. Thus $\gamma(X)=\bigcup_{A \in \operatorname{PC}^{\mathcal{C}}(X)} \gamma(A)$, in particular $X \in \operatorname{lnv}(\gamma)$ iff for every $A \in \operatorname{PC}^{\mathcal{C}}(X)$ we have $A \in \operatorname{lnv}(\gamma)$. Since $\gamma(X) \in \operatorname{lnv}(\gamma)$, for every $A \in \operatorname{PC}^{\mathcal{C}}(\gamma(X))$, we have both $A \in \operatorname{lnv}(\gamma)$ and $A \in \mathcal{C}$; it is then easily deduced that $\mathrm{PC}^{\mathcal{C} \cap \operatorname{lnv}(\gamma)}(X)=\mathrm{PC}^{\mathcal{C}}(\gamma(X))$, see Subsection 3.1 of [13] for a more detailed discussion. Hence $\operatorname{CS}^{\mathcal{C} \cap \ln v(\gamma)}=\operatorname{CS}^{\mathcal{C}} \mathrm{B}(\gamma)$, in particular $\mathrm{CS}^{\mathcal{C}} \mathrm{B}(\gamma)$ is an opening on on $\Pi^{*}(E)$.

This generalizes what we said previously about the partial connection $\mathcal{C}_{B}^{*}$ defined for $E=\mathbf{R}^{m}$ or $\mathbf{Z}^{m}$, with connection $\mathcal{C}_{\text {std }}$, and $B \in \mathcal{C}_{\text {std }} \backslash\{\emptyset\}$ : the $\mathcal{C}_{B}^{*}$ components of $X \in \mathcal{P}(E)$ are the $\mathcal{C}_{\text {std }}$-components of $\gamma_{B}(X)=X \circ B$. Indeed, here $\mathcal{C}_{B}^{*}=\mathcal{C}_{\text {std }} \cap \operatorname{lnv}\left(\gamma_{B}\right)$ and for any $p \in E$ we have $\delta_{B}(p)=B_{p} \in \mathcal{C}_{\text {std }}$.

We will now obtain an analogue for OC-thinnings of the opening $\mathrm{CS}^{\mathcal{C}} \mathrm{B}(\gamma)$. Recall from Subsection 2.1 that for an adjunction $(\varepsilon, \delta)$ and an antitone operator $\theta, \delta(\varepsilon \wedge \theta)$ is an OC-thinning, and that the family of OC-thinnings is a dual Moore family.

Proposition 8 Let $\mathcal{C}$ be a partial connection on $\mathcal{P}(E)$, and consider a family $\left\{\delta_{i}, \varepsilon_{i}, \theta_{i} \mid i \in I\right\}$ of operators on $\mathcal{P}(E)$, such that for every $i \in I, \theta_{i}$ is antitone, $\left(\varepsilon_{i}, \delta_{i}\right)$ is an adjunction on $\mathcal{P}(E)$, and for all $p \in E$ we have $\delta_{i}(p) \in \mathcal{C}$. Let $\kappa=\bigvee_{i \in I} \delta_{i}\left(\varepsilon_{i} \wedge \theta_{i}\right)$. Then $\mathrm{CS}^{\mathcal{C}} \mathrm{B}(\kappa)$ is an OC-thinning on $\Pi^{*}(E)$. 
Proof Let $\pi \in \Pi^{*}(E)$, and let $\pi_{0}=\operatorname{CS}^{\mathcal{C}} \mathrm{B}(\kappa)(\pi)$. By $(5,8)$,

$$
\pi_{0}=\bigcup_{C \in \mathrm{B}(\kappa)(\pi)} \mathrm{PC}^{\mathcal{C}}(C)=\bigcup\left\{\mathrm{PC}^{\mathcal{C}}(\kappa(B)) \mid B \in \pi, \kappa(B) \neq \emptyset\right\} .
$$

Thus the blocks of $\pi_{0}$ are the $\mathcal{C}$-components of $\kappa(B) \neq \emptyset$ for blocks $B$ of $\pi$. Now let $\pi_{1} \in \Pi^{*}(E)$ such that $\pi_{0} \leq \pi_{1} \leq \pi$. We will show that $\pi_{0} \leq \mathrm{CS}^{\mathcal{C}} \mathrm{B}(\kappa)\left(\pi_{1}\right)$. For this, we prove that for any $B \in \pi,\left[\pi_{0}\right]_{B} \leq \mathrm{B}(\kappa)\left(\left[\pi_{1}\right]_{B}\right)$.

Take any $B \in \pi$. If $\kappa(B)=\emptyset$, then $\left[\pi_{0}\right]_{B}=\emptyset \leq \mathrm{B}(\kappa)\left(\left[\pi_{1}\right]_{B}\right)$; we can thus assume that $\kappa(B) \neq \emptyset$, and then $\left[\pi_{0}\right]_{B}=\mathrm{PC}^{\mathcal{C}}(\kappa(B))$. Take $i \in I$. For $p \in \varepsilon_{i} \kappa(B)$ we have $\delta_{i}(p) \subseteq \kappa(B)$, and as $\delta_{i}(p) \in \mathcal{C}$, it must be included in one $\mathcal{C}$-component $C$ of $\kappa(B)$, and $p \in \varepsilon_{i}(C)$; thus

$$
\varepsilon_{i} \kappa(B)=\bigcup\left\{\varepsilon_{i}(C) \mid C \in \mathrm{PC}^{\mathcal{C}}(\kappa(B))\right\}
$$

Since $\mathrm{PC}^{\mathcal{C}}(\kappa(B))=\left[\pi_{0}\right]_{B} \leq\left[\pi_{1}\right]_{B}$, every $C \in \mathrm{PC}^{\mathcal{C}}(\kappa(B))$ is included in some $D \in\left[\pi_{1}\right]_{B}$, and as $\varepsilon_{i}$ is isotone, $\varepsilon_{i}(C) \subseteq \varepsilon_{i}(D)$, so we have

$$
\bigcup\left\{\varepsilon_{i}(C) \mid C \in \mathrm{PC}^{\mathcal{C}}(\kappa(B))\right\} \subseteq \bigcup\left\{\varepsilon_{i}(D) \mid D \in\left[\pi_{1}\right]_{B}\right\} .
$$

Now $\kappa=\bigvee_{j \in I} \delta_{j}\left(\varepsilon_{j} \wedge \theta_{j}\right) \geq \delta_{i}\left(\varepsilon_{i} \wedge \theta_{i}\right)$, and as $\varepsilon_{i}$ is isotone and $\varepsilon_{i} \delta_{i}$ is extensive, $\varepsilon_{i} \kappa \geq \varepsilon_{i} \delta_{i}\left(\varepsilon_{i} \wedge \theta_{i}\right) \geq \varepsilon_{i} \wedge \theta_{i}$. Summarizing these inequalities,

$$
\bigcup\left\{\varepsilon_{i}(D) \mid D \in\left[\pi_{1}\right]_{B}\right\} \supseteq \varepsilon_{i} \kappa(B) \supseteq\left(\varepsilon_{i} \wedge \theta_{i}\right)(B) .
$$

As $\left[\pi_{1}\right]_{B} \leq[\pi]_{B}=\mathbf{1}_{B}$, for every $D \in\left[\pi_{1}\right]_{B}$ we have $D \subseteq B$, and as $\theta_{i}$ is antitone, $\theta_{i}(D) \supseteq \theta_{i}(B)$. Hence

$$
\begin{gathered}
\bigcup\left\{\left(\varepsilon_{i} \wedge \theta_{i}\right)(D) \mid D \in\left[\pi_{1}\right]_{B}\right\}=\bigcup\left\{\varepsilon_{i}(D) \cap \theta_{i}(D) \mid D \in\left[\pi_{1}\right]_{B}\right\} \\
\supseteq \bigcup\left\{\varepsilon_{i}(D) \cap \theta_{i}(B) \mid D \in\left[\pi_{1}\right]_{B}\right\}=\left(\bigcup\left\{\varepsilon_{i}(D) \mid D \in\left[\pi_{1}\right]_{B}\right\}\right) \cap \theta_{i}(B) \\
\supseteq\left(\varepsilon_{i} \wedge \theta_{i}\right)(B) \cap \theta_{i}(B)=\left(\varepsilon_{i} \wedge \theta_{i} \wedge \theta_{i}\right)(B)=\left(\varepsilon_{i} \wedge \theta_{i}\right)(B) .
\end{gathered}
$$

Since $\delta_{i}$ is a dilation, we get

$$
\begin{gathered}
\bigcup\left\{\delta_{i}\left(\varepsilon_{i} \wedge \theta_{i}\right)(D) \mid D \in\left[\pi_{1}\right]_{B}\right\} \\
=\delta_{i}\left(\bigcup\left\{\left(\varepsilon_{i} \wedge \theta_{i}\right)(D) \mid D \in\left[\pi_{1}\right]_{B}\right\}\right) \supseteq \delta_{i}\left(\varepsilon_{i} \wedge \theta_{i}\right)(B) .
\end{gathered}
$$

Joining these inequalities for all $i \in I$, we obtain

$$
\begin{gathered}
\bigcup\left\{\kappa(D) \mid D \in\left[\pi_{1}\right]_{B}\right\}=\bigcup\left\{\bigcup_{i \in I} \delta_{i}\left(\varepsilon_{i} \wedge \theta_{i}\right)(D) \mid D \in\left[\pi_{1}\right]_{B}\right\} \\
\left.=\bigcup_{i \in I} \bigcup \delta_{i}\left(\varepsilon_{i} \wedge \theta_{i}\right)(D) \mid D \in\left[\pi_{1}\right]_{B}\right\} \supseteq \bigcup_{i \in I} \delta_{i}\left(\varepsilon_{i} \wedge \theta_{i}\right)(B)=\kappa(B) .
\end{gathered}
$$

It follows then that for any $C \in\left[\pi_{0}\right]_{B}=\mathrm{PC}^{\mathcal{C}}(\kappa(B)), C \subseteq \bigcup\{\kappa(D) \mid D \in$ $\left.\left[\pi_{1}\right]_{B}\right\}$. But as $\left[\pi_{0}\right]_{B} \leq\left[\pi_{1}\right]_{B}$, there is a unique $D_{C} \in\left[\pi_{1}\right]_{B}$ such that $C \subseteq D_{C}$, 
and for any other $D \in\left[\pi_{1}\right]_{B}$, we have $C \cap D=\emptyset$; since $\kappa$ is anti-extensive, we obtain $C \cap \kappa(D)=\emptyset$, and as $C \subseteq \bigcup\left\{\kappa(D) \mid D \in\left[\pi_{1}\right]_{B}\right\}$, we deduce that $C \subseteq \kappa\left(D_{C}\right)$, in particular $\kappa\left(D_{C}\right) \neq \emptyset$. This means that

$$
\left[\pi_{0}\right]_{B} \leq\left\{\kappa(D) \mid D \in\left[\pi_{1}\right]_{B}, \kappa(D) \neq \emptyset\right\}=\mathrm{B}(\kappa)\left(\left[\pi_{1}\right]_{B}\right) .
$$

As this inequality holds for all $B \in \pi$, by $(3,5)$ we obtain:

$$
\begin{gathered}
\operatorname{CS}^{\mathcal{C}} \mathrm{B}(\kappa)(\pi)=\pi_{0}=\bigvee_{B \in \pi}\left[\pi_{0}\right]_{B} \leq \bigvee_{B \in \pi} \mathrm{B}(\kappa)\left(\left[\pi_{1}\right]_{B}\right) \\
=\bigvee_{B \in \pi} \bigvee_{C \in\left[\pi_{1}\right]_{B}} \mathbf{1}_{\kappa(C)}=\bigvee_{C \in \bigcup_{B \in \pi}\left[\pi_{1}\right]_{B}}^{\mathbf{1}_{\kappa(C)}}=\bigvee_{C \in \pi_{1}} \mathbf{1}_{\kappa(C)}=\mathrm{B}(\kappa)\left(\pi_{1}\right) .
\end{gathered}
$$

But $\mathrm{CS}^{\mathcal{C}}$ is an opening, hence $\pi_{0}=\mathrm{CS}^{\mathcal{C}} \mathrm{B}(\kappa)(\pi) \leq \mathrm{CS}^{\mathcal{C}} \mathrm{B}(\kappa)\left(\pi_{1}\right)$. Thus $\mathrm{CS}^{\mathcal{C}} \mathrm{B}(\kappa)$ satisfies condition $(\star)$ of Lemma 2 , hence is an OC-thinning.

For example let $E=\mathbf{R}^{m}$ or $\mathbf{Z}^{m}$, and let $\mathcal{C}$ be a translation-invariant partial connection: $\forall A \in \mathcal{C}, \forall p \in E$, we have $A_{p} \in \mathcal{C}$; this holds for instance when $\mathcal{C}$ is the usual connection $\mathcal{C}_{\text {std }}$ (topological or arcwise connectivity for $\mathbf{R}^{m}$, connectivity based on a digital adjacency for $\mathbf{Z}^{m}$ ). Here the translation-invariant dilation $\delta_{A}$ by a structuring element $A$ satisfies the condition $\forall p \in E, \delta_{A}(p) \in \mathcal{C}$, iff $A \in \mathcal{C}$. By Matheron's decomposition theorem [7,18], a translation-invariant antitone operator on $\mathcal{P}(E)$ takes the form $X \mapsto \bigcup_{B \in \mathcal{B}}\left(X^{c} \ominus B\right)$ for a family $\mathcal{B}$ of structuring elements. Hence a translation-invariant operator $\kappa$ according to Proposition 8 takes the form

$$
\kappa(X)=\bigcup_{(A, B) \in \mathcal{V}}\left(\left[(X \ominus A) \cap\left(X^{c} \ominus B\right)\right] \oplus A\right),
$$

where $\mathcal{V} \subseteq \mathcal{C} \times \mathcal{P}(E)$; in other words, cf. (10), it is a union of generalized foreground openings with connected foreground structuring elements. In Figure 4 we illustrate Proposition 8 with $\kappa$ according to (12), for the extraction of block components whose width lies in a given interval.

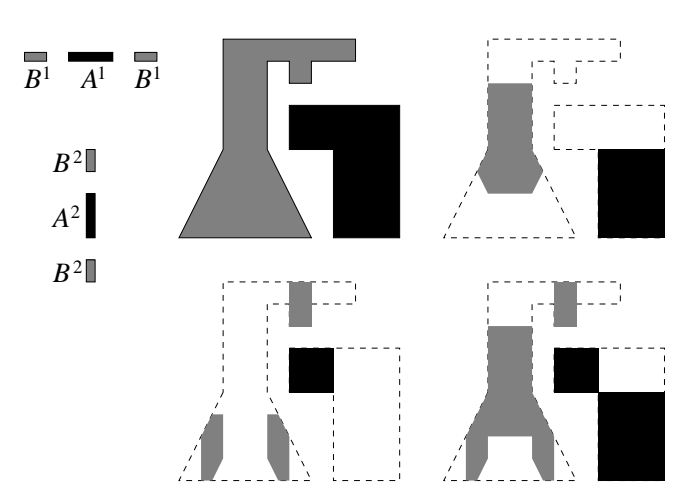

Fig. $4 E=\mathbf{Z}^{2}$. Top left: the foreground structuring elements $A_{1}, A_{2}$ and their corresponding background structuring elements $B_{1}, B_{2}$. Top middle: a partial partition with two blocks (in grey and black). Top right: apply the generalized foreground opening $(10)$ by $\left(A_{1}, B_{1}\right)$ to each block separately. Bottom middle: the same with $\left(A_{2}, B_{2}\right)$. Bottom right: in each block, decompose the union of the two generalized foreground openings into their connected components (two in the grey block, one in the black one). 
Generalized foreground openings (10) have been extended to grey-level images (i.e., numerical functions) $[12,8]$. In [9] they have been applied to the extraction of blood vessels in three-dimensional grey-level medical images. Vessels are modelled as narrow tubular objects in $E=\mathbf{Z}^{3}$, and one extracts them by a grey-level extension of (12), where for each $(A, B) \in \mathcal{V}, A$ is a sphere of varying radius and $B$ is a ring around $A$ (or a sampling of that ring), of varying orientation (say, normal to the 13 directions of the cube $\{-1,0,+1\}^{3}$ ); we illustrate in Figure 5 such a set $\mathcal{V}$ of pairs $(A, B)$.
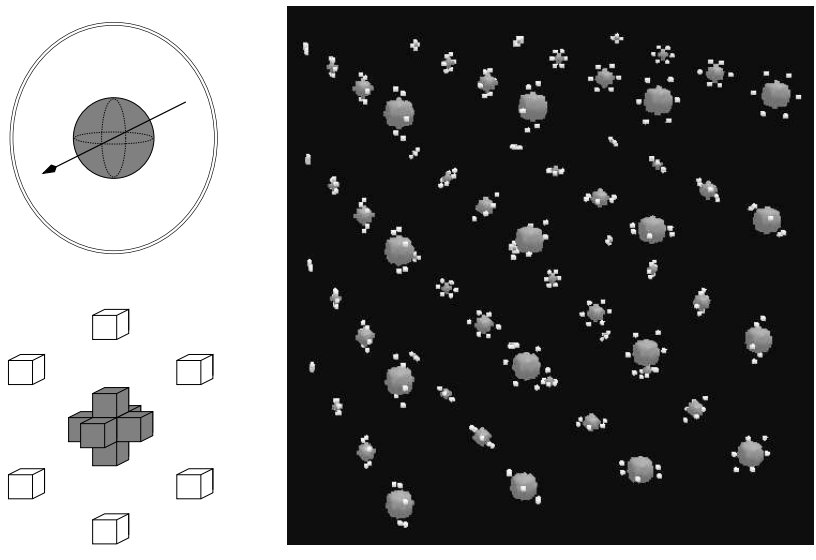

Fig. 5 (From [9].) Top left: a sphere $A$ surrounded by a ring $B$ in $\mathbf{R}^{3}$. Bottom left: their discrete version in $\mathbf{Z}^{3}$ Right: a series of pairs $(A, B)$ ( $A$ in grey, $B$ in white) of various sizes and orientations.

On the other hand, here we start from a binary image (not a grey-level one), apply $\kappa(12)$ to extract tubular objects, then decompose the resulting set into its connected components; by Proposition 8, the underlying operator on partial partitions is an OC-thinning, in particular it is idempotent.

We have described OC-thinnings on $\Pi^{*}(E)$ of the form $\mathrm{CS}^{\mathcal{C}} \mathrm{B}(\kappa)$ for a partial connection $\mathcal{C}$ and an OC-thinning $\kappa$ on $\mathcal{P}(E)$. In Subsection 3.3, we will obtain OC-thinnings taking the form $\mathrm{B}(\tau) \mathrm{CS}^{\mathcal{C}}$ for a trivial operator $\tau$ on $\mathcal{P}(E)$ (which is an OC-thinning, cf. Subsection 2.1).

\subsection{Residual combination of block splitting operators}

Serra [22] proposed to partition a set by combining a partial connection $\mathcal{C}_{1}$ and a connection $\mathcal{C}_{2}$. For every $X \in \mathcal{P}(E)$ one obtains the partial partition of its $\mathcal{C}_{1}$ components, and it is completed into a partition by adding to it the partition of $\mathcal{C}_{2}$-components of the residual of $\mathcal{C}_{1}$, that is, of the set of points of $X$ not covered by the $\mathcal{C}_{1}$-components of $X$. Thus we obtain the partition $\operatorname{PC}^{\mathcal{C}_{1}}(X) \cup$ $\mathrm{PC}^{\mathcal{C}_{2}}\left(X \backslash \operatorname{supp}\left[\mathrm{PC}^{\mathcal{C}_{1}}(X)\right]\right)$. This construction can be extended [13] by taking $n$ partial connections $\mathcal{C}_{1}, \ldots, \mathcal{C}_{n}$ and iteratively constructing partial partitions $\pi_{1}, \ldots, \pi_{n}$ of $X$ as follows: $\pi_{1}=\mathrm{PC}^{\mathcal{C}_{1}}(X)$, and for $i=2, \ldots, n$ set $\pi_{i}=\pi_{i-1} \cup$ $\mathrm{PC}^{\mathcal{C}_{i}}\left(X \backslash \operatorname{supp}\left[\pi_{i-1}\right]\right)$. Such a construction is involved in some segmentation algorithms, such as the multi-jump segmentation $[23,17,13]$. It can also be used 
in a multi-stage segmentation strategy [17], where a first partial segmentation of the image by a partially connective criterion extracts some objects, then a second segmentation of the remaining unsegmented background extracts further objects, etc.; for instance in a person's photograph, one first extracts the head, then the shoulders, etc. (see Figure 2.6 of [17]).

For example let $E=\mathbf{Z}^{m}$, take the connection $\mathcal{C}_{2}=\mathcal{C}_{\text {std }}$ (the usual connectivity), and the partial connection $\mathcal{C}_{1}=\mathcal{C}_{B}^{*}$ for a non-void $B \in \mathcal{C}_{s t d}$. Then for a set $X, \mathrm{PC}^{\mathcal{C}_{1}}(X) \cup \mathrm{PC}^{\mathcal{C}_{2}}\left(X \backslash \operatorname{supp}\left[\mathrm{PC}^{\mathcal{C}_{1}}(X)\right]\right)$ is the partition of $X$ into all $\mathcal{C}_{\text {std }}$-components of $X \circ B$ and of $X \backslash(X \circ B)$. This is illustrated (for $m=2$ ) in Figure 6 for a disk $B$ (a) and a bowtie-shaped set $X$ (b). Then by grouping these blocks into influence zones of the $\mathcal{C}_{B}^{*}$-components of $X(\mathrm{c})$, we obtain a segmentation of $X$ into its significant parts (d). Such a grouping of blocks into influence zones of the blocks of the first partial partition $\mathrm{PC}^{\mathcal{C}_{1}}(X)$ can also be applied in multi-stage image segmentation algorithms, such as the multi-jump.

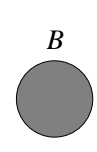

(a)

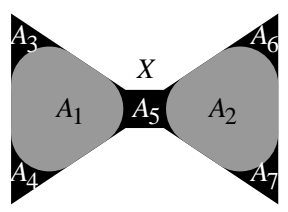

(b)

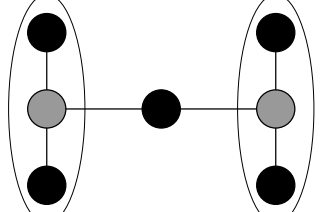

(c)

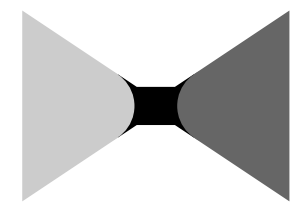

(d)

Fig. 6 Here $E=\mathbf{Z}^{2}$. (a) The structuring element $B \in \mathcal{C}_{\text {std }}$ is a disk. (b) The $\mathcal{C}_{B}^{*}$-components of the bowtie $X$ are the two $\mathcal{C}_{s t d}$-components $A_{1}, A_{2}$ of its opening $X \circ B$ (shown in grey). The residual $X \backslash(X \circ B)$ (shown in black) has $5 \mathcal{C}_{\text {std }}$-components $A_{3}, \ldots, A_{7}$. Thus $\mathrm{PC}_{B}^{*}(X) \cup$ $\mathrm{PC}^{\mathcal{C}_{\text {std }}}\left(X \backslash \operatorname{supp}\left[\mathrm{PC}^{\mathcal{C}_{B}^{*}}(X)\right]\right)$ is the partition of $X$ with the 7 blocks $A_{1}, \ldots, A_{7}$. (c) We build the adjacency graph of this partition, whose vertices are the 7 blocks $A_{i}$, and whose edges link vertices corresponding to adjacent blocks. The two ellipes represent the influence zones of the two vertices $A_{1}, A_{2}$ in the graph of $\mathrm{PC}^{\mathcal{C}_{B}^{*}}(X)$, namely the sets of vertices closer to one than to the other (according to the graph distance); note that $A_{5}$ is equidistant from both $A_{1}$ and $A_{2}$. (d) Fusing the blocks in the respective influence zones gives a final segmentation of $X$ into 3 blocks.

This multi-stage partitioning is similar to a method given in [10] for decomposing shapes in $E=\mathbf{Z}^{m}$ (or $\mathbf{R}^{m}$ ). Take $n$ structuring elements $B_{1}, \ldots, B_{n} \in$ $\mathcal{P}(E)$. Then for any $X \in \mathcal{P}(E)$ we construct the sequence of approximations $Y_{1}, \ldots, Y_{n} \in \mathcal{P}(E)$ by setting $Y_{1}=X \circ B_{1}$, and for $i=2, \ldots, n$, $Y_{i}=Y_{i-1} \cup\left(X \backslash Y_{i-1}\right) \circ B_{i}$. In [11] we showed (in the way of Proposition 4) that this construction yields a C-thinning.

We will similarly show that Serra's multi-stage partitioning leads to a block splitting C-thinning on $\Pi^{*}(E)$. This construction involves a binary operation on set splitting operators, that we call the residual combination; we will study it in detail. 
Definition 9 Let $\sigma, \theta$ be set splitting operators. The residual combination of $\sigma$ followed by $\theta$ is the set splitting operator $\operatorname{rc}[\sigma, \theta]$ defined by:

$$
\forall X \in \mathcal{P}(E), \quad \operatorname{rc}[\sigma, \theta](X)=\sigma(X) \cup \theta(X \backslash \operatorname{supp}[\sigma(X)])
$$

(NB. It is indeed the union of the two partial partitions $\sigma(X)$ and $\theta(X \backslash$ $\operatorname{supp}[\sigma(X)])$, because they have disjoint supports.) We define the residual combination of the succession of $n$ set splitting operators $\sigma_{1}, \ldots, \sigma_{n}(n \geq 1)$ recursively by

$$
\operatorname{rc}\left[\sigma_{1}\right]=\sigma_{1} \quad \text { and } \quad \operatorname{rc}\left[\sigma_{1}, \ldots, \sigma_{n}\right]=\operatorname{rc}\left[\operatorname{rc}\left[\sigma_{1}, \ldots, \sigma_{n-1}\right], \sigma_{n}\right](n>1)
$$

Thus Serra's construction is $\left.\mathrm{rc}^{\mathrm{P}} \mathrm{PC}^{\mathcal{C}_{1}}, \mathrm{PC}^{\mathcal{C}_{2}}\right]$. We illustrate in Figure 7 the residual combination of set shrinking operators derived from erosions by balls of varying size, then by squares of varying size, and finally from the openings by these squares.
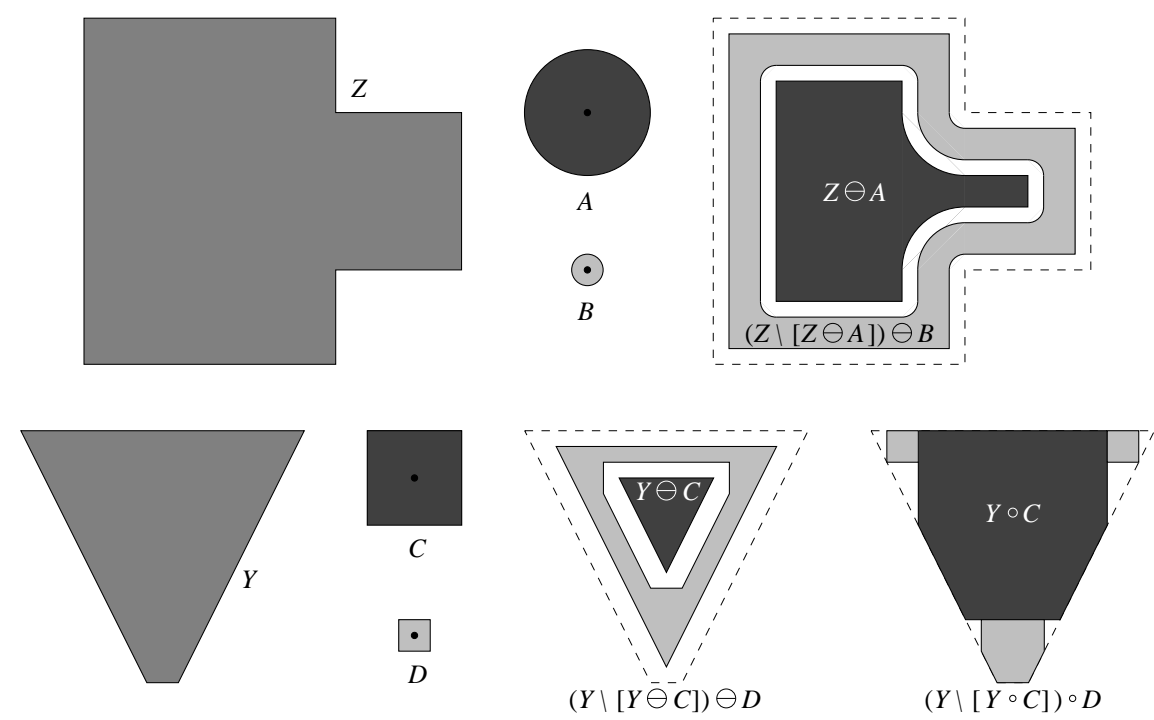

Fig. 7 Here $E=\mathbf{Z}^{2}$. Top left: a set $Z$ and two structuring elements $A$ and $B$. Top right: let $e_{A}$ and $e_{B}$ be the set shrinking operators derived from the erosions by $A$ and $B$ respectively, $e_{A}: X \mapsto \mathbf{1}_{X \ominus A}$ and $e_{B}: X \mapsto \mathbf{1}_{X \ominus B}$; we show $\operatorname{rc}\left[e_{A}, e_{B}\right](Z)=\{Z \ominus A,(Z \backslash[Z \ominus A]) \ominus B\}$. Bottom left: a set $Y$ and two structuring elements $C$ and $D$. Bottom middle: let $e_{C}$ and $e_{D}$ be the set shrinking operators derived from the erosions by $C$ and $D$ respectively, $e_{C}$ : $X \mapsto \mathbf{1}_{X \ominus C}$ and $e_{D}: X \mapsto \mathbf{1}_{X \ominus D}$; we show $\operatorname{rc}\left[e_{C}, e_{D}\right](Y)=\{Y \ominus C,(Y \backslash[Y \ominus C]) \ominus D\}$. Bottom right: let $g_{C}$ and $g_{D}$ be the set shrinking operators derived from the openings by $C$ and $D$ respectively, $g_{C}: X \mapsto \mathbf{1}_{X \circ C}$ and $g_{D}: X \mapsto \mathbf{1}_{X \circ D}$; we show $\operatorname{rc}\left[g_{C}, g_{D}\right](Y)=$ $\{Y \circ C,(Y \backslash[Y \circ C]) \circ D\}$. 
Proposition 10 Consider $n$ set splitting operators $\sigma_{1}, \ldots, \sigma_{n}(n \geq 2)$. For any $X \in \mathcal{P}(E)$, set

$$
\begin{aligned}
& X_{1}=X, Y_{1}=\operatorname{supp}\left[\sigma_{1}(X)\right], \\
& X_{i}=X \backslash \bigcup_{j=1}^{i-1} Y_{j}, Y_{i}=\operatorname{supp}\left[\sigma_{i}\left(X_{i}\right)\right] \quad(i=2, \ldots, n) ;
\end{aligned}
$$

then we have

$$
\operatorname{rc}\left[\sigma_{1}, \ldots, \sigma_{n}\right](X)=\bigcup_{i=1}^{n} \sigma_{i}\left(X_{i}\right) \text { and } \operatorname{supp}\left[\operatorname{rc}\left[\sigma_{1}, \ldots, \sigma_{n}\right](X)\right]=\bigcup_{i=1}^{n} Y_{i} .
$$

Furthermore, the residual combination is associative, for $1 \leq k<n$ we have:

$$
\operatorname{rc}\left[\operatorname{rc}\left[\sigma_{1}, \ldots, \sigma_{k}\right], \operatorname{rc}\left[\sigma_{k+1}, \ldots, \sigma_{n}\right]\right]=\operatorname{rc}\left[\sigma_{1}, \ldots, \sigma_{n}\right],
$$

in particular $\mathrm{rc}\left[\mathrm{rc}\left[\sigma_{1}, \sigma_{2}\right], \sigma_{3}\right]=\operatorname{rc}\left[\sigma_{1}, \operatorname{rc}\left[\sigma_{2}, \sigma_{3}\right]\right]$.

Proof We prove (16) by induction on $n$. For $n=1$ we have $\operatorname{rc}\left[\sigma_{1}\right](X)=$ $\sigma_{1}(X)=\sigma_{1}\left(X_{1}\right)$ and $\operatorname{supp}\left[\operatorname{rc}\left[\sigma_{1}\right](X)\right]=\operatorname{supp}\left[\sigma_{1}\left(X_{1}\right)\right]=Y_{1}$. Now for $n>1$, assuming (16) for $n-1$, we have:

$$
\begin{gathered}
\operatorname{rc}\left[\sigma_{1}, \ldots, \sigma_{n}\right](X)=\operatorname{rc}\left[\operatorname{rc}\left[\sigma_{1}, \ldots, \sigma_{n-1}\right], \sigma_{n}\right](X) \\
\left.=\operatorname{rc}\left[\sigma_{1}, \ldots, \sigma_{n-1}\right](X) \cup \sigma_{n}\left(X \backslash \operatorname{supp}\left[\operatorname{rc}\left[\sigma_{1}, \ldots, \sigma_{n-1}\right](X)\right]\right]\right) \\
=\left(\bigcup_{i=1}^{n-1} \sigma_{i}\left(X_{i}\right)\right) \cup \sigma_{n}\left(X \backslash \bigcup_{i=1}^{n-1} Y_{i}\right)=\left(\bigcup_{i=1}^{n-1} \sigma_{i}\left(X_{i}\right)\right) \cup \sigma_{n}\left(X_{n}\right)=\bigcup_{i=1}^{n} \sigma_{i}\left(X_{i}\right) .
\end{gathered}
$$

We get then

$$
\operatorname{supp}\left[\operatorname{rc}\left[\sigma_{1}, \ldots, \sigma_{n}\right](X)\right]=\operatorname{supp}\left[\bigcup_{i=1}^{n} \sigma_{i}\left(X_{i}\right)\right]=\bigcup_{i=1}^{n} \operatorname{supp}\left[\sigma_{i}\left(X_{i}\right)\right]=\bigcup_{i=1}^{n} Y_{i}
$$

Now the associativity. We have

$$
\operatorname{rc}\left[\sigma_{1}, \ldots, \sigma_{k}\right](X)=\bigcup_{i=1}^{k} \sigma_{i}\left(X_{i}\right) \text { and } \operatorname{supp}\left[\operatorname{rc}\left[\sigma_{1}, \ldots, \sigma_{k}\right](X)\right]=\bigcup_{i=1}^{k} Y_{i} .
$$

Hence

$$
\begin{gathered}
\operatorname{rc}\left[\operatorname{rc}\left[\sigma_{1}, \ldots, \sigma_{k}\right], \operatorname{rc}\left[\sigma_{k+1}, \ldots, \sigma_{n}\right]\right](X) \\
=\bigcup_{i=1}^{k} \sigma_{i}\left(X_{i}\right) \cup \operatorname{rc}\left[\sigma_{k+1}, \ldots, \sigma_{n}\right]\left(X \backslash \bigcup_{i=1}^{k} Y_{i}\right) \\
=\bigcup_{i=1}^{k} \sigma_{i}\left(X_{i}\right) \cup \operatorname{rc}\left[\sigma_{k+1}, \ldots, \sigma_{n}\right]\left(X_{k+1}\right)=\bigcup_{i=1}^{k} \sigma_{i}\left(X_{i}\right) \cup \bigcup_{i=k+1}^{n} \sigma_{i}\left(\bar{X}_{i}\right),
\end{gathered}
$$


where $\bar{X}=X_{k+1}$ and the $\bar{X}_{i}$ are obtained by (15) with the initial set $\bar{X}$ instead of $X$, and the sequence $k+1, \ldots, n$ instead of $1, \ldots, n$. Thus we have

$$
\begin{array}{rlrl}
\bar{X}_{k+1} & =\bar{X}, \bar{Y}_{k+1}=\operatorname{supp}\left[\sigma_{k+1}(\bar{X})\right] \\
\bar{X}_{i} & =\bar{X} \backslash \bigcup_{j=k+1}^{i-1} \bar{Y}_{j}, \bar{Y}_{i} & =\operatorname{supp}\left[\sigma_{i}\left(\bar{X}_{i}\right)\right] \quad(i=k+2, \ldots, n) .
\end{array}
$$

We show by induction that $\bar{X}_{i}=X_{i}$ and $\bar{Y}_{i}=Y_{i}$. Since $\bar{X}=X_{k+1}$, this is true for $i=k+1$. Now for $i>k+1$, assuming that the result is true for all $j=k+1, \ldots, i-1$, we have

$$
\begin{gathered}
\bar{X}_{i}=\bar{X} \backslash \bigcup_{j=k+1}^{i-1} \bar{Y}_{j}=X_{k+1} \backslash \bigcup_{j=k+1}^{i-1} Y_{j} \\
=\left(X \backslash \bigcup_{j=1}^{k} Y_{j}\right) \backslash \bigcup_{j=k+1}^{i-1} Y_{j}=X \backslash \bigcup_{j=1}^{i-1} Y_{j}=X_{i},
\end{gathered}
$$

and $\bar{Y}_{i}=\operatorname{supp}\left[\sigma_{i}\left(\bar{X}_{i}\right)\right]=\operatorname{supp}\left[\sigma_{i}\left(X_{i}\right)\right]=Y_{i}$. Therefore

$$
\begin{gathered}
\operatorname{rc}\left[\operatorname{rc}\left[\sigma_{1}, \ldots, \sigma_{k}\right], \operatorname{rc}\left[\sigma_{k+1}, \ldots, \sigma_{n}\right]\right](X) \\
=\bigcup_{i=1}^{k} \sigma_{i}\left(X_{i}\right) \cup \bigcup_{i=k+1}^{n} \sigma_{i}\left(X_{i}\right)=\bigcup_{i=1}^{n} \sigma_{i}\left(X_{i}\right)=\operatorname{rc}\left[\sigma_{1}, \ldots, \sigma_{n}\right](X) .
\end{gathered}
$$

In particular for $n=3$, setting $k=1$, we get

$$
\operatorname{rc}\left[\sigma_{1}, \operatorname{rc}\left[\sigma_{2}, \sigma_{3}\right]\right]=\operatorname{rc}\left[\sigma_{1}, \sigma_{2}, \sigma_{3}\right]=\operatorname{rc}\left[\operatorname{rc}\left[\sigma_{1}, \sigma_{2}\right], \sigma_{3}\right] .
$$

We illustrate in Figure 8 the construction (15) for $i=1,2,3$.


Fig. 8 From left to right:

$X_{1}=X ; \pi_{1}=\sigma_{1}\left(X_{1}\right)$ and $X_{2}=X_{1} \backslash \operatorname{supp}\left[\pi_{1}\right]$; $\pi_{2}=\sigma_{2}\left(X_{2}\right)$ and $X_{3}=X_{2}$ $\operatorname{supp}\left[\pi_{2}\right]=X_{1} \backslash \operatorname{supp}\left[\pi_{1} \cup \pi_{2}\right]$; $\pi_{3}=\sigma_{3}\left(X_{3}\right)$ and $X_{4}=X_{3}$ $\operatorname{supp}\left[\pi_{3}\right]=X_{1} \backslash \operatorname{supp}\left[\pi_{1} \cup \pi_{2} \cup\right.$ $\left.\pi_{3}\right]$.

Proposition 11 Let the binary operations + and - on $\Pi^{*}(E)$ be partially defined as follows for $\pi^{1}, \pi^{2} \in \Pi^{*}(E)$ : 
- If $\pi^{1} \geq \pi^{2}$, then $\pi^{1}-\pi^{2}$ is defined by

$$
\pi^{1}-\pi^{2}=\left\{B \backslash \operatorname{supp}\left(\left[\pi^{2}\right]_{B}\right) \mid B \in \pi^{1}, B \backslash \operatorname{supp}\left(\left[\pi^{2}\right]_{B}\right) \neq \emptyset\right\} .
$$

In other words, we remove from every block of $\pi^{1}$ all blocks of $\pi^{2}$ that it contains, and keep non-empty resulting blocks.

- If $\operatorname{supp}\left(\pi^{1}\right) \cap \operatorname{supp}\left(\pi^{2}\right)=\emptyset$, then $\pi^{1}+\pi^{2}$ is defined by

$$
\pi^{1}+\pi^{2}=\pi^{1} \vee \pi^{2}=\pi^{1} \cup \pi^{2} .
$$

See Figure 9. Then:

1. For $\pi^{1}, \pi^{2} \in \Pi^{*}(E)$ with $\pi^{1} \geq \pi^{2}$,

$$
\pi^{1}-\pi^{2}=\pi^{1} \wedge \mathbf{1}_{E \backslash \operatorname{supp}\left(\pi^{2}\right)}=\left\{B \backslash \operatorname{supp}\left(\pi^{2}\right) \mid B \in \pi^{1}, B \backslash \operatorname{supp}\left(\pi^{2}\right) \neq \emptyset\right\}
$$

and

$$
\operatorname{supp}\left(\pi^{1}-\pi^{2}\right)=\operatorname{supp}\left(\pi^{1}\right) \backslash \operatorname{supp}\left(\pi^{2}\right) .
$$

2. The operations + and - satisfy the conditions $(a, b, c, d, e, f)$ of Proposition 4 .

3. Given set splitting operators $\sigma, \theta$, for any $\pi \in \Pi^{*}(E)$ we have

$$
\beta(\operatorname{rc}[\sigma, \theta])(\pi)=\beta(\sigma)(\pi)+\beta(\theta)(\pi-\beta(\sigma)(\pi)) .
$$
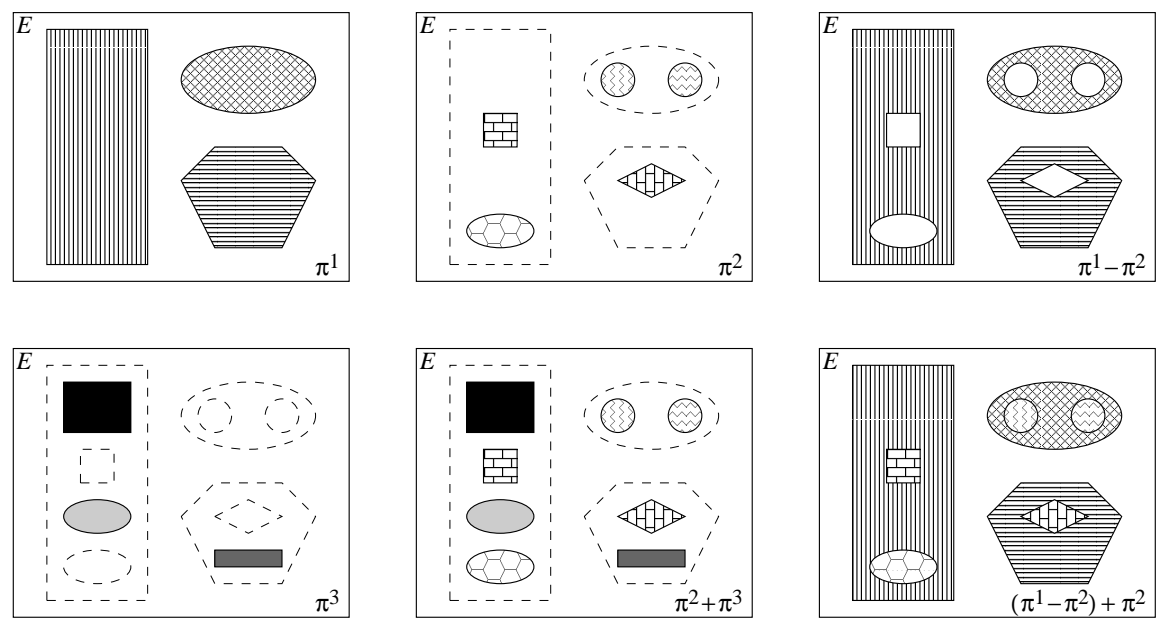

Fig. 9 Here $E=\mathbf{Z}^{2}$. In all partial partitions, each block is identified by a distinctive greylevel or hatching. Top left: $\pi^{1} \in \Pi^{*}(E)$. Top center: $\pi^{2} \leq \pi^{1}$. Top right: $\pi^{1}-\pi^{2}$. Bottom left: $\pi^{3} \leq \pi^{1}-\pi^{2}$. Bottom center: $\pi^{2}+\pi^{3}$. Bottom right $\left(\pi^{1}-\pi^{2}\right)+\pi^{2} \leq \pi^{1}$. 
Proof 1. Let $B \in \pi^{1}$. For any other $C \in \pi^{1}$, we have $\operatorname{supp}\left(\left[\pi^{2}\right]_{C}\right) \subseteq C$ and $B \cap C=\emptyset$, so $B$ is disjoint from $\operatorname{supp}\left(\left[\pi^{2}\right]_{C}\right)$. By $(3), \operatorname{supp}\left(\pi^{2}\right)=$ $\bigcup_{C \in \pi^{1}} \operatorname{supp}\left(\left[\pi^{2}\right]_{C}\right)$. Hence

$$
B \backslash \operatorname{supp}\left(\pi^{2}\right)=B \backslash\left[\bigcup_{C \in \pi^{1}} \operatorname{supp}\left(\left[\pi^{2}\right]_{C}\right)\right]=B \backslash \operatorname{supp}\left(\left[\pi^{2}\right]_{B}\right) .
$$

Thus $\pi^{1}-\pi^{2}=\left\{B \backslash \operatorname{supp}\left(\pi^{2}\right) \mid B \in \pi^{1}, B \backslash \operatorname{supp}\left(\pi^{2}\right) \neq \emptyset\right\}$. Now

$$
\mathbf{1}_{B} \wedge \mathbf{1}_{E \backslash \operatorname{supp}\left(\pi^{2}\right)}=\mathbf{1}_{B \cap\left(E \backslash \operatorname{supp}\left(\pi^{2}\right)\right)}=\mathbf{1}_{B \backslash \operatorname{supp}\left(\pi^{2}\right)},
$$

which is $\left\{B \backslash \operatorname{supp}\left(\pi^{2}\right)\right\}$ if $B \backslash \operatorname{supp}\left(\pi^{2}\right) \neq \emptyset$, and $\emptyset$ otherwise. Hence

$$
\pi^{1}-\pi^{2}=\bigcup_{B \in \pi^{1}}\left(\mathbf{1}_{B} \wedge \mathbf{1}_{E \backslash \operatorname{supp}\left(\pi^{2}\right)}\right)=\bigvee_{B \in \pi^{1}}\left(\mathbf{1}_{B} \wedge \mathbf{1}_{E \backslash \operatorname{supp}\left(\pi^{2}\right)}\right)
$$

By (2), we get thus

$$
\pi^{1}-\pi^{2}=\left(\bigvee_{B \in \pi^{1}} \mathbf{1}_{B}\right) \wedge \mathbf{1}_{E \backslash \operatorname{supp}\left(\pi^{2}\right)}=\pi^{1} \wedge \mathbf{1}_{E \backslash \operatorname{supp}\left(\pi^{2}\right)} .
$$

Then

$$
\begin{gathered}
\operatorname{supp}\left(\pi^{1}-\pi^{2}\right)=\operatorname{supp}\left(\pi^{1} \wedge \mathbf{1}_{E \backslash \operatorname{supp}\left(\pi^{2}\right)}\right)=\operatorname{supp}\left(\pi^{1}\right) \cap \operatorname{supp}\left(\mathbf{1}_{E \backslash \operatorname{supp}\left(\pi^{2}\right)}\right) \\
=\operatorname{supp}\left(\pi^{1}\right) \cap\left(E \backslash \operatorname{supp}\left(\pi^{2}\right)\right)=\operatorname{supp}\left(\pi^{1}\right) \backslash \operatorname{supp}\left(\pi^{2}\right) .
\end{gathered}
$$

2. Let us check each condition of Proposition 4. Let $\pi^{w}, \pi^{x}, \pi^{y}, \pi^{z} \in$ $\Pi^{*}(E)$ taking the role of $w, x, y, z$ in the statement of Proposition 4.

(a) For $\pi^{y} \leq \pi^{x}, \pi^{x}-\pi^{y}$ is defined.

(b) If $\pi^{y} \leq \pi^{x} \leq \pi^{w}$, then we have

$$
\pi^{x}-\pi^{y}=\pi^{x} \wedge \mathbf{1}_{E \backslash \operatorname{supp}\left(\pi^{y}\right)} \leq \pi^{w} \wedge \mathbf{1}_{E \backslash \operatorname{supp}\left(\pi^{y}\right)}=\pi^{w}-\pi^{y} .
$$

(c) For $\pi^{y} \leq \pi^{x}$ and $\pi^{z} \leq \pi^{x}-\pi^{y}$, we have $\operatorname{supp}\left(\pi^{z}\right) \subseteq \operatorname{supp}\left(\pi^{x}-\pi^{y}\right)=$ $\operatorname{supp}\left(\pi^{x}\right) \backslash \operatorname{supp}\left(\pi^{y}\right)$, hence $\operatorname{supp}\left(\pi^{z}\right) \cap \operatorname{supp}\left(\pi^{y}\right)=\emptyset$, so $\pi^{y}+\pi^{z}$ is defined.

(d) Since $\pi^{y} \leq \pi^{x}$ and

$$
\pi^{z} \leq \pi^{x}-\pi^{y}=\pi^{x} \wedge \mathbf{1}_{E \backslash \operatorname{supp}\left(\pi^{y}\right)} \leq \pi^{x},
$$

we have $\pi^{y}+\pi^{z}=\pi^{y} \vee \pi^{z} \leq \pi^{x}$.

(e) Obviously $\pi^{y} \leq \pi^{y} \vee \pi^{z}=\pi^{y}+\pi^{z}$.

(f) For $B \in \pi^{y}$ we have $B \subseteq \operatorname{supp}\left(\pi^{y}\right)$, so $B \backslash \operatorname{supp}\left(\pi^{y}\right)=\emptyset$, while for $B \in \pi^{z}$, we have $B \cap \operatorname{supp}\left(\pi^{y}\right)=\emptyset$, so $B \backslash \operatorname{supp}\left(\pi^{y}\right)=B$. Hence

$$
\begin{aligned}
\left(\pi^{y}+\pi^{z}\right)-\pi^{y}= & \left(\pi^{y} \cup \pi^{z}\right)-\pi^{y} \\
= & \left\{B \backslash \operatorname{supp}\left(\pi^{y}\right) \mid B \in \pi^{y} \cup \pi^{z}, B \backslash \operatorname{supp}\left(\pi^{y}\right) \neq \emptyset\right\} \\
= & \left\{B \backslash \operatorname{supp}\left(\pi^{y}\right) \mid B \in \pi^{y}, B \backslash \operatorname{supp}\left(\pi^{y}\right) \neq \emptyset\right\} \\
& \cup\left\{B \backslash \operatorname{supp}\left(\pi^{y}\right) \mid B \in \pi^{z}, B \backslash \operatorname{supp}\left(\pi^{y}\right) \neq \emptyset\right\} \\
= & \emptyset \cup\left\{B \mid B \in \pi^{z}\right\}=\pi^{z} .
\end{aligned}
$$


3. Let $\pi \in \Pi^{*}(E)$. Combining (13) with (4), we get

$$
\begin{aligned}
\beta(\operatorname{rc}[\sigma, \theta])(\pi) & =\bigcup_{B \in \pi} \operatorname{rc}[\sigma, \theta](B)=\bigcup_{B \in \pi}[\sigma(B) \cup \theta(B \backslash \operatorname{supp}[\sigma(B)])] \\
& =\left(\bigcup_{B \in \pi} \sigma(B)\right) \cup\left(\bigcup_{B \in \pi} \theta(B \backslash \operatorname{supp}[\sigma(B)])\right) \\
& =\beta(\sigma)(\pi) \cup\left(\bigcup_{B \in \pi} \theta(B \backslash \operatorname{supp}[\sigma(B)])\right) .
\end{aligned}
$$

Now $\sigma(B)=[\beta(\sigma)(\pi)]_{B}$, and $\theta(\emptyset)=\varnothing$ is redundant in this union, so we get:

$$
\begin{gathered}
\beta(\operatorname{rc}[\sigma, \theta])(\pi)=\beta(\sigma)(\pi) \cup \bigcup_{B \in \pi} \theta\left(B \backslash \operatorname{supp}\left([\beta(\sigma)(\pi)]_{B}\right)\right)= \\
\beta(\sigma)(\pi) \cup \bigcup\left\{\theta\left(B \backslash \operatorname{supp}\left([\beta(\sigma)(\pi)]_{B}\right)\right) \mid B \in \pi, B \backslash \operatorname{supp}\left([\beta(\sigma)(\pi)]_{B}\right) \neq \emptyset\right\} \\
=\beta(\sigma)(\pi) \cup \bigcup\{\theta(C) \mid C \in \pi-\beta(\sigma)(\pi)\}=\beta(\sigma)(\pi) \cup \beta(\theta)(\pi-\beta(\sigma)(\pi)) .
\end{gathered}
$$

Clearly $\beta(\sigma)(\pi)$ and $\beta(\theta)(\pi-\beta(\sigma)(\pi))$ have disjoint supports, thus the above union is $\mathrm{a}+$, and the result follows.

Corollary 12 1. Given two set splitting operators $\sigma, \theta$ such that $\beta(\sigma)$ and $\beta(\theta)$ are $C$-thinnings, $\beta(\mathrm{rc}[\sigma, \theta])$ is a C-thinning. More precisely, given $\pi, \pi^{\prime} \in \Pi^{*}(E)$ such that $\beta(\operatorname{rc}[\sigma, \theta])(\pi) \leq \pi^{\prime} \leq \pi$, we have $\beta(\sigma)\left(\pi^{\prime}\right)=$ $\beta(\sigma)(\pi)$ and $\beta(\theta)\left(\pi^{\prime}-\beta(\sigma)\left(\pi^{\prime}\right)\right)=\beta(\theta)(\pi-\beta(\sigma)(\pi))$.

2. Given $n$ partial connections $\mathcal{C}_{1}, \ldots, \mathcal{C}_{n}(n \geq 1), \beta\left(\operatorname{rc}\left[\mathrm{PC}^{\mathcal{C}_{1}}, \ldots, \mathrm{PC}^{\mathcal{C}_{n}}\right]\right)$ is a $C$-thinning. More precisely, given $\pi, \pi^{\prime} \in \Pi^{*}(E)$ such that

$$
\beta\left(\mathrm{rC}\left[\mathrm{PC}^{\mathcal{C}_{1}}, \ldots, \mathrm{PC}^{\mathcal{C}_{n}}\right]\right)(\pi) \leq \pi^{\prime} \leq \pi
$$

for $m=1, \ldots, n$ we have

$$
\beta\left(\operatorname{rc}\left[\mathrm{PC}^{\mathcal{C}_{1}}, \ldots, \mathrm{PC}^{\mathcal{C}_{m}}\right]\right)\left(\pi^{\prime}\right)=\beta\left(\operatorname{rc}\left[\mathrm{PC}^{\mathcal{C}_{1}}, \ldots, \mathrm{PC}^{\mathcal{C}_{m}}\right]\right)(\pi) .
$$

Proof Item 1 follows by combining Proposition 11 (items 2 and 3) with Proposition 4 (taking $x=\pi, y=\pi^{\prime}, \eta=\beta(\sigma)$ and $\zeta=\beta(\theta)$ ). Now each $\mathrm{CS}^{\mathcal{C}_{i}}=$ $\beta\left(\mathrm{PC}^{\mathcal{C}_{i}}\right)$ is an opening (hence a C-thinning) on $\Pi^{*}(E)$, so following (14), item 2 is obtained from item 1 by induction on $n$.

Remark 13 Given a set $U$ of labels, a labelled partial partition is a pair $(\pi, F)$, where $\pi \in \Pi^{*}(E)$ and $F$ is a map $\pi \rightarrow U$; alternately, it is a subset $\lambda$ of $(\mathcal{P}(E) \backslash\{\emptyset\}) \times U$ such that for any two distinct $(B, u),(C, v) \in \lambda$, we have $B \cap C=\emptyset$. They appear in image classification, where from a grey-level image one extracts different objects together with a description or set of attributes for each object. The labels can be semantic descriptions (for instance in remote sensing: "water", "forest", "urban area", "agricultural land", etc.), numerical values (measuring some physical features) or vectors (corresponding to several measurements). 
Let $\Pi_{U}^{*}(E)$ be the set of labelled partial partitions. When $U$ is a poset, $\Pi_{U}^{*}(E)$ is partially ordered by $\left(\pi_{1}, F_{1}\right) \leq\left(\pi_{2}, F_{2}\right)$ iff $\pi_{1} \leq \pi_{2}$ and $\forall p \in$ $\operatorname{supp}\left(\pi_{1}\right), F_{1}\left(\mathrm{Cl}_{\pi_{1}}(p)\right) \leq F_{2}\left(\mathrm{Cl}_{\pi_{2}}(p)\right)$; in other words, for any $B \in \pi_{1}$, there is some $C \in \pi_{2}$ such that $B \subseteq C$ and $F_{1}(B) \leq F_{2}(C)$. When $U$ is a complete lattice, $\Pi_{U}^{*}(E)$ is also a complete lattice. It is then easy to extend the operations + and - to $\Pi_{U}^{*}(E)$, and items 1 and 2 of Proposition 11 will hold. We can also extend block splitting operators to $\Pi_{U}^{*}(E)$, and then item 3 of Proposition 11 and item 1 of Corollary 12 will hold.

\subsection{Block selection and constrained connectivity}

We will now consider operators of the form $\mathrm{B}(\tau) \mathrm{CS}^{\mathcal{C}}$ for a trivial operator $\tau$ (cf. Subsection 2.1) and a partial connection $\mathcal{C}$, then suprema of such operators; they will be OC-thinnings. Our results constitute a mathematical basis for Soille's approach to image segmentation [27-30], see Figure 10. We give an example of their possible use in shape decomposition. We end this subsection by a brief discussion of operators on sets called attribute thinnings [4] (or anti-extensive grain operators [6]), which also enter into our framework.

Theorem 14 Let $\mathrm{p}$ be a predicate on $\mathcal{P}(E) \backslash\{\emptyset\}$, and let $\tau_{\mathrm{p}}$ be the trivial operator on $\mathcal{P}(E)$ corresponding to $\mathrm{p}$. Then:

1. for any $\pi \in \Pi^{*}(E)$,

$$
\mathrm{B}\left(\tau_{\mathrm{p}}\right)(\pi)=\{B \in \pi \mid \mathrm{p}(B)=1\},
$$

and for any $p \in E$,

$$
\mathrm{Cl}_{\mathrm{B}\left(\tau_{\mathrm{p}}\right)(\pi)}(p)=\tau_{\mathrm{p}}\left(\mathrm{Cl}_{\pi}(p)\right) ;
$$

2. $\mathrm{B}\left(\tau_{\mathrm{p}}\right)$ is an $O C$-thinning;

3. for any idempotent block splitting operator $\beta(\sigma)$, we have $\beta(\sigma) \mathrm{B}\left(\tau_{\mathrm{p}}\right) \beta(\sigma)$ $=\mathrm{B}\left(\tau_{\mathrm{p}}\right) \beta(\sigma)$, and $\mathrm{B}\left(\tau_{\mathrm{p}}\right) \beta(\sigma)$ is block splitting and idempotent;

4. given a partial connection $\mathcal{C}, \mathrm{B}\left(\tau_{\mathrm{p}}\right) \mathrm{CS}^{\mathcal{C}}$ is a block splitting $O C$-thinning.

Proof 1. By $(5), \mathrm{B}\left(\tau_{\mathrm{p}}\right)(\pi)=\left\{\tau_{\mathrm{p}}(B) \mid B \in \pi, \tau_{\mathrm{p}}(B) \neq \emptyset\right\}$. But $\tau_{\mathrm{p}}(B)=$ $\emptyset$ for $\mathrm{p}(B)=0$, while $\tau_{\mathrm{p}}(B)=B$ for $\mathrm{p}(B)=1$. This gives (17). By (6), either $p \in \tau_{\mathrm{p}}\left(\mathrm{Cl}_{\pi}(p)\right)$ and $\mathrm{Cl}_{\mathrm{B}\left(\tau_{\mathrm{p}}\right)(\pi)}(p)=\tau_{\mathrm{p}}\left(\mathrm{Cl}_{\pi}(p)\right)$, or $p \notin \tau_{\mathrm{p}}\left(\mathrm{Cl}_{\pi}(p)\right)$ and $\mathrm{Cl}_{\mathrm{B}\left(\tau_{\mathrm{p}}\right)(\pi)}(p)=\emptyset$. But $p \notin \tau_{\mathrm{p}}\left(\mathrm{Cl}_{\pi}(p)\right)$ means that either $\mathrm{Cl}_{\pi}(p)=\emptyset$, or $\mathrm{Cl}_{\pi}(p) \neq$ $\emptyset$ and $\mathrm{p}\left(\mathrm{Cl}_{\pi}(p)\right)=0$, both cases giving $\tau_{\mathrm{p}}\left(\mathrm{Cl}_{\pi}(p)\right)=\emptyset$. Thus (18) holds in any case.

2. By Proposition $6, \tau_{p}$ is an OC-thinning. Hence $\mathrm{B}\left(\tau_{\mathrm{p}}\right)$ is overcondensing by Proposition 7 . By $(17), \mathrm{B}\left(\tau_{\mathrm{p}}\right)$ is idempotent. Obviously $\mathrm{B}\left(\tau_{\mathrm{p}}\right)$ is antiextensive.

3. As $\beta(\sigma)$ is idempotent, for any $\pi \in \Pi^{*}(E), \beta(\sigma)(\pi) \in \operatorname{lnv}(\beta(\sigma))$. By (7), this means that $\beta(\sigma)(\pi) \in \Pi^{*}(E, \mathcal{F}(\sigma))$, in other words every block of $\beta(\sigma)(\pi)$ belongs to $\mathcal{F}(\sigma)$. By (17), the blocks of $\mathrm{B}\left(\tau_{\mathrm{p}}\right) \beta(\sigma)(\pi)$ are some of the blocks of $\beta(\sigma)(\pi)$, in $\mathcal{F}(\sigma)$, so $\mathrm{B}\left(\tau_{\mathrm{p}}\right) \beta(\sigma)(\pi) \in \Pi^{*}(E, \mathcal{F}(\sigma))=\operatorname{lnv}(\beta(\sigma))$. 
This implies that $\beta(\sigma) \mathrm{B}\left(\tau_{\mathbf{p}}\right) \beta(\sigma)(\pi)=\mathrm{B}\left(\tau_{\mathbf{p}}\right) \beta(\sigma)(\pi)$. Thus $\beta(\sigma) \mathrm{B}\left(\tau_{\mathbf{p}}\right) \beta(\sigma)=$ $\mathrm{B}\left(\tau_{\mathbf{p}}\right) \beta(\sigma)$, and we deduce that

$$
\left(\mathrm{B}\left(\tau_{\mathbf{p}}\right) \beta(\sigma)\right)^{2}=\mathrm{B}\left(\tau_{\mathbf{p}}\right) \beta(\sigma) \mathrm{B}\left(\tau_{\mathbf{p}}\right) \beta(\sigma)=\mathrm{B}\left(\tau_{\mathbf{p}}\right) \mathrm{B}\left(\tau_{\mathbf{p}}\right) \beta(\sigma)=\mathrm{B}\left(\tau_{\mathbf{p}}\right) \beta(\sigma),
$$

because $\mathrm{B}\left(\tau_{\mathrm{p}}\right)$ is idempotent (as OC-thinning). Therefore $\mathrm{B}\left(\tau_{\mathrm{p}}\right) \beta(\sigma)$ is idempotent. Being a composition of block splitting operators, it is block splitting.

4. $\mathrm{CS}^{\mathcal{C}}$ is an opening; then item 3 gives $\mathrm{CS}^{C} \mathrm{~B}\left(\tau_{\mathrm{p}}\right) \mathrm{CS}^{C}=\mathrm{B}\left(\tau_{\mathrm{p}}\right) \mathrm{CS}^{C}$; by item $2, \mathrm{~B}\left(\tau_{\mathrm{p}}\right)$ is an OC-thinning. Then by item 1 of Proposition $3, \mathrm{~B}\left(\tau_{\mathrm{p}}\right) \mathrm{CS}^{C}$ is an OC-thinning. It is block splitting by item 3 .

Corollary 15 Associate to each $i \in I \neq \emptyset$ a trivial operator $\tau^{i}$ and a partial connection $\mathcal{C}^{i}$. Let $\kappa=\bigvee_{i \in I} \mathrm{~B}\left(\tau^{i}\right) \mathrm{CS}^{\mathcal{C}^{i}}$; then $\kappa$ is a block splitting OC-thinning.

Furthermore, if $I$ is totally ordered (a chain), and $\mathcal{C}^{i}$ increases with $i(i<$ $\left.j \Rightarrow \mathcal{C}^{i} \subseteq \mathcal{C}^{j}\right)$, given the system of partial connection openings $\left(\gamma_{p}^{i}, p \in E\right)$ of $\mathcal{C}^{i}$, we have:

$$
\forall \pi \in \Pi^{*}(E), \forall p \in E, \quad \mathrm{Cl}_{\kappa(\pi)}(p)=\bigcup_{i \in I} \tau^{i} \gamma_{p}^{i}\left(\mathrm{Cl}_{\pi}(p)\right) .
$$

If in addition I is finite, $\mathrm{Cl}_{\kappa(\pi)}(p)$ is the greatest among the $\tau^{i} \gamma_{p}^{i}\left(\mathrm{Cl}_{\pi}(p)\right), i \in I$.

Proof By item 4 of Theorem 14, $\kappa$ is a supremum of OC-thinnings, hence it is an OC-thinning. Still by item $4, \kappa$ is a supremum of block splitting operators, it is thus block splitting.

Now let $I$ be a chain with $i<j \Rightarrow \mathcal{C}^{i} \subseteq \mathcal{C}^{j}$. Combining (9) with (18), we obtain for every $i \in I, \pi \in \Pi^{*}(E)$ and $p \in E$ :

$$
\mathrm{Cl}_{\mathrm{B}\left(\tau^{i}\right) \operatorname{CS}^{i}(\pi)}(p)=\tau^{i}\left(\mathrm{Cl}_{\mathrm{CS}^{C^{i}}}(\pi)(p)\right)=\tau^{i} \gamma_{p}^{i}\left(\mathrm{Cl}_{\pi}(p)\right) .
$$

Let $i, j \in I$, where $i \neq j$. If one of $\tau^{i} \gamma_{p}^{i}\left(\mathrm{Cl}_{\pi}(p)\right)$ or $\tau^{j} \gamma_{p}^{j}\left(\mathrm{Cl}_{\pi}(p)\right)$ is empty, it is included in the other. Suppose now that $\tau^{i} \gamma_{p}^{i}\left(\mathrm{Cl}_{\pi}(p)\right) \neq \emptyset \neq \tau^{j} \gamma_{p}^{j}\left(\mathrm{Cl}_{\pi}(p)\right)$; we have $i<j$ or $j<i$. For $i<j$, as $\mathcal{C}^{i} \subseteq \mathcal{C}^{j}$, we have $\gamma_{p}^{i}\left(\mathrm{Cl}_{\pi}(p)\right) \subseteq \gamma_{p}^{j}\left(\mathrm{Cl}_{\pi}(p)\right)$, hence $\tau^{i} \gamma_{p}^{i}\left(\mathrm{Cl}_{\pi}(p)\right)=\gamma_{p}^{i}\left(\mathrm{Cl}_{\pi}(p)\right) \subseteq \gamma_{p}^{j}\left(\mathrm{Cl}_{\pi}(p)\right)=\tau^{j} \gamma_{p}^{j}\left(\mathrm{Cl}_{\pi}(p)\right)$. For $j<i$ we have the reverse inclusion. Thus the set

$$
\left\{\tau^{i} \gamma_{p}^{i}\left(\mathrm{Cl}_{\pi}(p)\right) \mid i \in I\right\}=\left\{\mathrm{Cl}_{\mathrm{B}\left(\tau^{i}\right) \mathrm{CS}^{C^{i}(\pi)}}(p) \mid i \in I\right\}
$$

is totally ordered, hence it is directed, and by (1):

$$
\mathrm{Cl}_{\kappa(\pi)}(p)=\mathrm{Cl}_{\bigvee_{i \in I} \mathrm{~B}\left(\tau^{i}\right) \operatorname{CS}^{C^{i}(\pi)}}(p)=\bigcup_{i \in I} \mathrm{Cl}_{\mathrm{B}\left(\tau^{i}\right) \operatorname{CS}^{C^{i}(\pi)}}(p)=\bigcup_{i \in I} \tau^{i} \gamma_{p}^{i}\left(\mathrm{Cl}_{\pi}(p)\right)
$$

that is (19). When $I$ is finite, the union of the finite chain of all $\tau^{i} \gamma_{p}^{i}\left(\mathrm{Cl}_{\pi}(p)\right)$, $i \in I$, is the greatest one among them. 
Note that although (1) requires only a directed family of point classes, the above argument does not extend to the case where the set of partial connections $\mathcal{C}^{i}(i \in I)$ is directed instead of totally ordered. Indeed, if $\mathcal{C}^{i}, \mathcal{C}^{j} \subseteq \mathcal{C}^{k}$, we may have $\tau^{i} \gamma_{p}^{i}\left(\mathrm{Cl}_{\pi}(p)\right)=\gamma_{p}^{i}\left(\mathrm{Cl}_{\pi}(p)\right)$ and $\tau^{j} \gamma_{p}^{j}\left(\mathrm{Cl}_{\pi}(p)\right)=\gamma_{p}^{j}\left(\mathrm{Cl}_{\pi}(p)\right)$, but $\tau^{k} \gamma_{p}^{k}\left(\mathrm{Cl}_{\pi}(p)\right)=\emptyset$, so the set of $\tau^{i} \gamma_{p}^{i}\left(\mathrm{Cl}_{\pi}(p)\right)$ for $i \in I$ is not necessarily directed.

We can easily deal with the case of an isotone predicate:

Proposition 16 Let $\mathrm{p}$ be an isotone predicate on $\mathcal{P}(E) \backslash\{\emptyset\}$, and let $\mathcal{C}_{\mathrm{p}}=$ $\{\emptyset\} \cup\{X \in \mathcal{P}(E) \backslash\{\emptyset\} \mid \mathrm{p}(X)=1\}$. Then:

1. $\mathcal{C}_{\mathrm{p}}$ is a partial connection, and $\mathrm{B}\left(\tau_{\mathrm{p}}\right)=\mathrm{CS}^{\mathcal{C}_{\mathrm{p}}}$;

2. for any partial connection $\mathcal{C}, \mathrm{B}\left(\tau_{\mathrm{p}}\right) \mathrm{CS}^{\mathcal{C}}=\mathrm{CS}^{\mathcal{C}_{\mathrm{p}} \cap \mathcal{C}}$.

Proof 1. As explained at the end of Subsection 2.1, $\mathcal{C}_{\mathrm{p}}=\operatorname{Inv}\left(\tau_{\mathrm{p}}\right)$, and it is a dual Moore family, hence a partial connection. For any $X \in \mathcal{P}(E) \backslash\{\emptyset\}$, either $\mathrm{p}(X)=1$ and $X \in \mathcal{C}_{\mathrm{p}}$, in other words $X$ is its unique $\mathcal{C}_{\mathrm{p}}$-component, or $\mathrm{p}(X)=0$ and then, as $\mathrm{p}$ is isotone, for any $Y \in \mathcal{P}(X)$ such that $Y \neq \emptyset$ we have $Y \notin \mathcal{C}_{\mathrm{p}}$, in other words $X$ has no $\mathcal{C}_{\mathrm{p}}$-component. Thus $\operatorname{PC}^{\mathcal{C}_{\mathrm{p}}}(X)=\mathbf{1}_{\tau_{\mathrm{p}}(X)}$ (and this remains true for $\left.X=\emptyset: \mathrm{PC}^{\mathcal{C}_{\mathrm{p}}}(\emptyset)=\emptyset=\mathbf{1}_{\emptyset}=\mathbf{1}_{\tau_{\mathrm{p}}(\emptyset)}\right)$. Hence $\mathrm{PC}^{\mathcal{C}_{\mathrm{p}}}=\mathbf{1}_{\tau_{\mathrm{p}}}$, from which we deduce that $\mathrm{CS}^{\mathcal{C}_{\mathrm{p}}}=\beta\left(\mathrm{PC}^{\mathcal{C}_{\mathrm{p}}}\right)=\beta\left(\mathbf{1}_{\tau_{\mathrm{p}}}\right)=\mathrm{B}\left(\tau_{\mathrm{p}}\right)$.

2. Let us first show that for $X \in \mathcal{P}(E)$, the $\mathcal{C}_{\mathrm{p}} \cap \mathcal{C}$-components of $X$ are exactly the $\mathcal{C}$-components $C$ of $X$ for which $\mathrm{p}(C)=1$. Let $C$ be a $\mathcal{C}_{\mathrm{p}} \cap \mathcal{C}$ component of $X$; then $C \neq \emptyset$ and $C \in \mathcal{C}_{\mathrm{p}} \cap \mathcal{C}$, so $\mathrm{p}(C)=1$; if $C$ was not a $\mathcal{C}$-component of $X$, there would be $C^{\prime} \in \mathcal{C}$ with $C \subset C^{\prime} \subseteq X$, and as $\mathrm{p}$ is isotone, we would have $\mathrm{p}\left(C^{\prime}\right)=1$, hence $C^{\prime} \in \mathcal{C}_{\mathrm{p}} \cap \mathcal{C}$, contradicting the fact that $C$ is a $\mathcal{C}_{\mathrm{p}} \cap \mathcal{C}$-component of $X$; therefore $C$ is a $\mathcal{C}$-component of $X$ and $\mathrm{p}(C)=1$. Conversely, let $C$ be a $\mathcal{C}$-component of $X$ such that $\mathrm{p}(C)=1$; then $C \in \mathcal{C}_{\mathrm{p}}$, so $C \in \mathcal{C}_{\mathrm{p}} \cap \mathcal{C}$; if $C$ was not a $\mathcal{C}_{\mathrm{p}} \cap \mathcal{C}$-component of $X$, there would be $C^{\prime} \in \mathcal{C}_{\mathrm{p}} \cap \mathcal{C}$ with $C \subset C^{\prime} \subseteq X$, so $C^{\prime} \in \mathcal{C}$, contradicting the fact that $C$ is a $\mathcal{C}$-component of $X$; therefore $C$ is a $\mathcal{C}_{\mathrm{p}} \cap \mathcal{C}$-component of $X$.

For any $\pi \in \Pi^{*}(E)$, the blocks of $\operatorname{CS}^{\mathcal{C}}(\pi)$ are all $\mathcal{C}$-components of blocks of $\pi$; then the blocks of $\mathrm{B}\left(\tau_{\mathrm{p}}\right) \mathrm{CS}^{\mathcal{C}}(\pi)$ are all $\mathcal{C}$-components $C$ of blocks of $\pi$ such that $\mathrm{p}(C)=1$; in other words, they are all $\mathcal{C}_{\mathrm{p}} \cap \mathcal{C}$-components of blocks of $\pi$, that is, the blocks of $\operatorname{CS}^{\mathcal{C}_{\mathrm{p}} \cap \mathcal{C}}(\pi)$. Hence $\mathrm{B}\left(\tau_{\mathrm{p}}\right) \operatorname{CS}^{\mathcal{C}}(\pi)=\operatorname{CS}^{\mathcal{C}_{\mathrm{p}} \cap \mathcal{C}}(\pi)$.

For a shorter but more abstract proof of item 2 , we remark that $\mathrm{B}\left(\tau_{\mathrm{p}}\right) \mathrm{CS}^{\mathcal{C}}=$ $\mathrm{CS}^{\mathcal{C}_{\mathrm{p}}} \mathrm{CS}^{\mathcal{C}}$ is an opening, hence it must be the infimum of $\mathrm{CS}^{\mathcal{C}_{\mathrm{p}}}$ and $\mathrm{CS}^{\mathcal{C}}$ in the complete lattice of openings; by the isomorphism between the two complete lattices of partial connections and of block splitting openings, it must correspond to the infimum of $\mathcal{C}_{\mathrm{p}}$ and $\mathcal{C}$ in the complete lattice of partial connections, that is $\mathcal{C}_{\mathrm{p}} \cap \mathcal{C}$, so $\mathrm{B}\left(\tau_{\mathrm{p}}\right) \mathrm{CS}^{\mathcal{C}}=\mathrm{CS}^{\mathcal{C}_{\mathrm{p}} \cap \mathcal{C}}$.

Since $\mathrm{B}\left(\tau_{\mathrm{p}}\right)$ acts on a partial partition by selecting all blocks $B$ with $\mathrm{p}(B)=$ 1 , cf. (17), it is easy to decompose it when $p$ is a combination of predicates. The following result is straigtforward, we leave its proof to the reader: 
Proposition 17 Consider a non-void family $\mathrm{p}_{i}, i \in I \neq \emptyset$, of predicates on $\mathcal{P}(E) \backslash\{\emptyset\}$. Then for any $\pi \in \Pi^{*}(E)$,

$$
\mathrm{B}\left(\tau \bigvee_{i \in I} \mathrm{p}_{i}\right)(\pi)=\bigcup_{i \in I} \mathrm{~B}\left(\tau_{\mathrm{p}_{i}}\right)(\pi)=\bigvee_{i \in I} \mathrm{~B}\left(\tau_{\mathrm{p}_{i}}\right)(\pi)
$$

and

$$
\mathrm{B}\left(\tau_{\bigwedge_{i \in I} \mathrm{p}_{i}}\right)(\pi)=\bigcap_{i \in I} \mathrm{~B}\left(\tau_{\mathrm{p}_{i}}\right)(\pi)=\bigwedge_{i \in I} \mathrm{~B}\left(\tau_{\mathrm{p}_{i}}\right)(\pi) .
$$

When $I$ is finite, $I=\{1, \ldots, n\}$,

$$
\mathrm{B}\left(\tau \bigwedge_{i=1}^{n} \mathrm{p}_{i}\right)=\prod_{i=1}^{n} \mathrm{~B}\left(\tau_{\mathrm{p}_{i}}\right)
$$

Soille's approach to image segmentation [27-30] follows the model of Corollary 15 . Here $I=\{0, \ldots, n\}$, and given a numerical function $F: E \rightarrow T$ and connective criteria $\mathrm{cr}_{i}(i=0, \ldots, n)$, we take the connections $\mathcal{C}^{i}=\mathcal{C}_{\mathrm{cr}_{i}}^{F}=\{A \in$ $\left.\mathcal{P}(E) \mid \mathrm{Cr}_{i}[F, A]=1\right\}$; we suppose that the $\mathrm{cr}_{i}$ increase with $i$, so do the $\mathcal{C}^{i}$. Now each $\tau^{i}$ is guided by a "constraining" criterion (i.e., $\tau^{i}(B)=1$ iff $[F, B]$ satisfies that criterion). In fact, $\tau^{0}$ preserves $\mathcal{C}^{0}$, so $\kappa(\pi) \geq \mathrm{CS}^{\mathcal{C}^{0}}(\pi)$, hence for $\pi \in \Pi(E)$ we have $\kappa(\pi) \in \Pi(E)$. The segmentation of the function $F$ is then given by the partition $\kappa\left(\mathbf{1}_{E}\right)$. We illustrate it in Figure 10 for a onedimensional image, with the "connected Lipschitz" segmentation criterion and the "bounded total variation" constraining predicate.

A similar approach can be adopted for the decomposition of a shape into its significant parts. Here we asssume that a growing sequence $\mathcal{C}^{1}, \ldots, \mathcal{C}^{n}$ of (partial) connections and a predicate $\mathrm{p}$ are given independently of any numerical function. Let us illustrate this with an example.

Let $E=\mathbf{Z}^{m}$, with a "standard" connection $\mathcal{C}_{s t d}$. For any radius $r>0$, let $B_{r}$ be the closed ball or radius $r$ centered about the origin. Let $\mathcal{C}_{r}$ be the set of all $X \in \mathcal{C}_{s t d}$ such that $X \circ B_{r}=X$ (i.e., all $\mathcal{C}_{s t d}$-connected unions of translates of $B_{r}$ ). Then $\mathcal{C}_{r}=\mathcal{C}_{B_{r}}^{*}$ according to our notation, it is a partial connection, and for any $X \in \mathcal{P}(E)$, the $\mathcal{C}_{r}$-components of $X$ are the $\mathcal{C}_{s t d^{-}}$ components of $X \circ B_{r}$ (see Figure 6). For $r>s>0$, as $B_{r}$ is a union of translates of $B_{s}$, for every $X \in \mathcal{P}(E)$ we have $X \circ B_{r} \subseteq X \circ B_{s}$, hence $\mathcal{C}_{r} \subseteq \mathcal{C}_{s}$. We take thus a strictly decreasing sequence of radii $r_{1}>\cdots>r_{n}>0$ (for example $r_{i}=2^{n-i} r$ for some $r>0$ ), and set $\mathcal{C}^{i}=\mathcal{C}_{r_{i}}$; thus $\mathcal{C}^{1} \subseteq \ldots \subseteq \mathcal{C}^{n}$. We define the predicate $\mathrm{p}$ by $\mathrm{p}(X)=1$ iff for each $i=1, \ldots, n, X$ has at most one $\mathcal{C}^{i}$-component, that is, $X \circ B_{r_{i}}$ is either empty or $\mathcal{C}_{s t d}$-connected. We can interpret $\mathrm{p}$ by saying that $\mathrm{p}(X)=1$ iff $X$ "consists of one piece". This is illustrated (for $m=2$ ) in Figure 11, where the set appearing to have two pieces does not satisfy predicate $\mathrm{p}$. We see then in Figure 12 that by applying the segmentation operator $\kappa=\bigvee_{i=1}^{n} \mathrm{~B}\left(\tau_{\mathrm{p}}\right) \mathrm{CS}^{\mathcal{C}^{i}}$ to $\mathbf{1}_{X}$, the shape with two pieces is segmented into two blocks corresponding to them, while the shapes appearing in one piece have a segmentation with one block. Of course, the segmentation 




Fig. 10 Here $E=T=\mathbf{Z}$. For each $s \geq 0$, let lip ${ }^{s}$ be the criterion given by $\operatorname{lip}^{s}[F, A]=1$ if $A$ is connected and $F$ is is Lipschitz with slope $s$ on $A$; this criterion is connective. Top: a function $F: E \rightarrow T$; it leads to the connections $\mathcal{C}_{\text {lip }}^{F}$. Next row: we choose a height $u>0$, and define the constraining predicate $\mathrm{p}$ by $\mathrm{p}(A)=1$ iff $\sup _{x, y \in A}|F(x)-F(y)| \leq u$. Non-singletons blocks $B$ are shown in light grey when $\mathrm{p}(B)=1$ and in dark grey when $\mathrm{p}(B)=0$; hatchings denote rows of singleton blocks (they always satify $\mathrm{p}(B)=1$ ). Next rows in descending order: the segmentations $\mathrm{PC}^{\mathcal{C}_{\text {lip }}^{F}}(E)$ for $s=0,0.5,1,2$. Bottom: the final segmentation is the partition spanned by all blocks $B$ from the 4 segmentations having $\mathrm{p}(B)=1$.
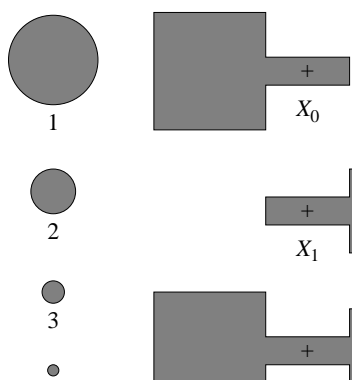

3
3
4

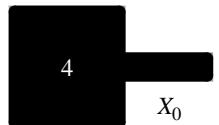

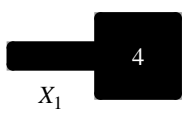



Fig. 11 Here $E=\mathbf{Z}^{2}$. Left: the 4 balls $B_{r_{1}}, \ldots, B_{r_{4}}$, with radii $r_{i}$ forming a geometric sequence of ratio $1 / 2$. Center: the three sets $X_{0}, X_{1}$ and $X_{2}\left(X_{2}=X_{0} \cup X_{1}\right)$, where the + indicates the position of the origin. Right: the graph of the function $i \mapsto$ $\left|\mathrm{PC}^{\mathcal{C}^{i}}\left(X_{j}\right)\right|=\mid \mathrm{PC}^{\mathcal{C}}$ std $\left(X_{j} \circ B_{r_{i}}\right) \mid$ for $j=0,1,2$. Thus $\mathrm{p}\left(X_{0}\right)=\mathrm{p}\left(X_{1}\right)=1$ but $\mathrm{p}\left(X_{2}\right)=0$.

Fig. 12 Left to center: over the three sets $X_{j}(j=0,1,2)$ in grey, we show in black the segmentation partial partition $\kappa\left(\mathbf{1}_{X_{j}}\right)=\bigvee_{i=1}^{4} \mathrm{~B}\left(\tau_{\mathrm{p}}\right) \mathrm{CS}^{\mathcal{C}^{i}}\left(\mathbf{1}_{X_{j}}\right)=\bigvee_{i=1}^{4} \mathrm{~B}\left(\tau_{\mathrm{p}}\right)\left(\mathrm{PC}^{\mathcal{C}^{i}}\left(X_{j}\right)\right)$; each block is a $\mathcal{C}^{i}$-component of $X_{j}$, we indicate that value $i$ on it (in white). Now $\mathrm{p}\left(X_{0}\right)=$ $\mathrm{p}\left(X_{1}\right)=1$ and the segmentation of $X_{0}$ and $X_{1}$ has a unique block, while $\mathrm{p}\left(X_{2}\right)=0$ and the segmentation of $X_{2}$ has two blocks. Right: a partial partition $\left\{X_{3}, X_{4}\right\}$ of $X_{2}$, here $\mathrm{p}\left(X_{3}\right)=\mathrm{p}\left(X_{4}\right)=1$ and $\kappa\left(\left\{X_{3}, X_{4}\right\}\right)>\kappa\left(\mathbf{1}_{X_{2}}\right)$. 
does not cover the whole shape, in particular the corners are rounded, but it is possible to recover these corners by the method illustrated in Figure 6 .

It should be noted that the result shown in Figure 12 cannot be obtained by partitioning the shape into its connected components according to some partial connection. Indeed, we have here $X_{2}=X_{0} \cup X_{1}$, and the unique block $Y_{0}$ in the segmentation of $X_{0}$ intersects $Y_{1}$, the one of $X_{1}$, so if they were connected components, $Y_{0}$ and $Y_{1}$ would both join inside a same connected component of $X_{2}$, which is not the case. Also, at the right of Figure 12 we have $\kappa\left(\left\{X_{2}\right\}\right)<\left\{X_{3}, X_{4}\right\}<\left\{X_{2}\right\}$ but $\kappa\left(\left\{X_{3}, X_{4}\right\}\right)>\kappa\left(\left\{X_{2}\right\}\right)$, so $\kappa$ is not a C-thinning.

Let us end this section with two general remarks on possible extensions of the scope of our results.

Remark 18 We have studied block splitting openings, C-thinnings and OCthinnings on $\Pi^{*}(E)$. We can extend them to $\Pi(E)$, thanks to a method introduced in [15]. There we defined the inclusion map

$$
I N: \Pi(E) \rightarrow \Pi^{*}(E): \pi \mapsto \pi,
$$

and the map that fills a partial partition by singleton blocks outside its support:

$$
F S: \Pi^{*}(E) \rightarrow \Pi(E): \pi \mapsto \pi \cup \mathbf{0}_{E \backslash \operatorname{supp}(\pi)}=\pi \vee \mathbf{0}_{E}
$$

Then we showed that $(I N, F S)$ is an adjunction $\Pi(E) \rightleftharpoons \Pi^{*}(E)$. Given $\kappa$ : $\Pi^{*}(E) \rightarrow \Pi^{*}(E)$, consider:

$$
F S \kappa I N: \Pi(E) \rightarrow \Pi(E): \pi \mapsto F S(\kappa(\pi))=\kappa(\pi) \vee \mathbf{0}_{E} .
$$

Since $(I N, F S)$ is an adjunction, by item 2 of Proposition 3 (and the wellknown similar result for openings), we obtain that $F S \kappa I N$ will inherit from $\kappa$ the property of being respectively an opening / a C-thinning / an OCthinning.

Furthermore, if $\kappa$ is a block splitting operator, $\kappa=\beta(\sigma)$, then $F S \kappa I N$ will also be block splitting, namely $F S \kappa I N=\beta\left(\sigma^{\prime}\right)$ for $\sigma^{\prime}: X \mapsto \sigma(X) \vee \mathbf{0}_{X}$; indeed, for any $\pi \in \Pi(E)$ we have:

$$
\begin{gathered}
\beta\left(\sigma^{\prime}\right)(\pi)=\bigvee_{B \in \pi} \sigma^{\prime}(B)=\bigvee_{B \in \pi}\left(\sigma(B) \vee \mathbf{0}_{B}\right)=\left(\bigvee_{B \in \pi} \sigma(B)\right) \vee\left(\bigvee_{B \in \pi} \mathbf{0}_{B}\right) \\
=\left(\bigvee_{B \in \pi} \sigma(B)\right) \vee \mathbf{0}_{E}=\beta(\sigma)(\pi) \vee \mathbf{0}_{E}=(F S \beta(\sigma) I N)(\pi) .
\end{gathered}
$$

If $\kappa=\mathrm{CS}^{\mathcal{C}}$ for a partial connection $\mathcal{C}$, then $F S \kappa I N=\mathrm{CS}^{\mathcal{C}^{\prime}}$, where $\mathcal{C}^{\prime}=$ $\mathcal{C} \cup \mathcal{S}(E)$, the least connection containing $\mathcal{C}$.

For example in the segmentation of Figure 12, if we want to have a partition instead of a partial partition, we replace the partial connection $\mathcal{C}^{i}$ by the connection $\mathcal{C}^{i} \cup \mathcal{S}(E)$; the resulting segmentation of $X$ will then be $\kappa\left(\mathbf{1}_{X}\right) \vee \mathbf{0}_{X}$ instead of $\kappa\left(\mathbf{1}_{X}\right)$, in other words the parts of the set $X$ not covered by the partial partition $\kappa\left(\mathbf{1}_{X}\right)$ will be covered by singletons. 
Remark 19 In mathematical morphology, given a connection $\mathcal{C}$ and a predicate $\mathrm{p}$, one defines an attribute thinning [4] as the operator that removes from a set $X$ all $\mathcal{C}$-components $Z$ such that $\mathrm{p}(Z)=0$; formally, the result is written $\bigcup_{x \in X} \tau_{\mathrm{p}}\left(\gamma_{x}(X)\right)$. When the predicate $\mathrm{p}$ is isotone, this operator will be an opening, it is then called an attribute opening. This is the anti-extensive case of the so-called grain operators according to [6] (quite generally, such an operator can also add to $X$ all $\mathcal{C}$-components of $X^{c}$ that do not satisfy some predicate q). Of courses, everything works also if we take for $\mathcal{C}$ a partial connection.

In our framework, this operator can be written:

$$
\theta_{\mathrm{p}}^{\mathcal{C}}: X \mapsto \operatorname{supp}\left[\mathrm{B}\left(\tau_{\mathrm{p}}\right)\left(\mathrm{PC}^{\mathcal{C}}(X)\right)\right]=\operatorname{supp}\left[\mathrm{B}\left(\tau_{\mathrm{p}}\right) \mathrm{CS}^{\mathcal{C}}\left(\mathbf{1}_{X}\right)\right]
$$

in other words, $\theta_{\mathrm{p}}^{\mathcal{C}}=\operatorname{supp} \cdot \mathrm{B}\left(\tau_{\mathrm{p}}\right) \mathrm{CS}^{\mathcal{C}} \cdot \mathbf{1}_{\bullet}$. Since $\left(\mathbf{1}_{\bullet}\right.$, supp) is an adjunction and $\mathrm{B}\left(\tau_{\mathrm{p}}\right) \mathrm{CS}^{\mathcal{C}}$ is an OC-thinning (by Theorem 14 ), the attribute thinning $\theta_{\mathrm{p}}^{\mathcal{C}}$ will be an OC-thinning (by item 2 of Proposition 3). When the predicate $p$ is isotone, $\theta_{\mathrm{p}}^{\mathcal{C}}$ is an opening; in fact we can use Proposition 16: $\theta_{\mathrm{p}}^{\mathcal{C}}(X)$ is the union of all $\left(\mathcal{C}_{\mathrm{p}} \cap \mathcal{C}\right)$-components of $X$, where

$$
\mathcal{C}_{\mathrm{p}} \cap \mathcal{C}=\{\emptyset\} \cup\{X \in \mathcal{C} \backslash\{\emptyset\} \mid \mathrm{p}(X)=1\}
$$

We can be more general: for any anti-extensive operator $\psi$ on partial partitions, define the operator on sets $\bar{\psi}=\operatorname{supp} \cdot \psi \cdot \mathbf{1}_{\bullet}: X \mapsto \operatorname{supp}\left[\psi\left(1_{X}\right)\right]$. Since supp is a complete morphism [15]), the map $\psi \mapsto \bar{\psi}$ is a complete morphism. Also, by item 2 of Proposition 3, whenever $\psi$ is respectively an opening / a C-thinning / an OC-thinning, $\bar{\psi}$ will have that property.

\section{Discussion and conclusion}

The analysis of block splitting openings in the first paper, together with [13], provides a theoretical basis for Serra's approach to segmentation, that we have called connective segmentation $[13,17]$.

Then Section 3 expands our analysis by building idempotent block splitting operators that are not isotone; this is achieved by combining block splitting openings with other constructions. We rely on the background of Subsection 2.1. In Subsection 3.1, one should mainly remember Proposition 8, because it shows how operators like those used in practical applications for extracting tubular shapes $[8,9]$, lead to block splitting OC-thinnings on partial partitions. Subsection 3.2 shows that Serra's [22] residual combination of block splitting openings is analogous to the residual combination of set openings considered in [11], and has the same property of being a C-thinning; this approach is used in the so-called composed segmentation of [17]. Subsection 3.3 shows how the composition of block splitting into $\mathcal{C}$-components and block selection according to predicates, leads to OC-thinnings. It springs from the work of Soille [27-30] on segmentation by the combination of connective and constraining criteria. We call his approach constrained connective segmentation; we have thus given 
here its theoretical basis. Besides image segmentation, our results can also be applied to the decomposition of shapes, cf. Figures 1, 11 and 12.

Our approach considers only block splitting operators, and emphasizes conditions for their idempotence, because this corresponds to current views on image segmentation [28]. Indeed, we explained in the introduction of the first paper that the segmentation of an image $E \rightarrow T$ according to a homogeneity criterion, means that one associates to $F$ a set splitting operator $\sigma$ that splits every set $X$ into a partial partition $\sigma(X)$ of $X$, whose blocks are homogeneous for $F$ according to the criterion; furthermore, it was required that $\beta(\sigma)$ is idempotent and that for every $\pi_{0}, \pi \in \Pi^{*}(E)$, we have

$$
\beta(\sigma)\left(\pi_{0}\right)<\pi \leq \pi_{0} \Longrightarrow \beta(\sigma)(\pi)<\pi
$$

in other words, $\beta(\sigma)\left(\pi_{0}\right)$ is a maximal element of the set of all invariants of $\beta(\sigma)$ that are $\leq \pi_{0}$. When $\beta(\sigma)$ is a C-thinning (in particular, if it is an opening), we have $\beta(\sigma)\left(\pi_{0}\right) \leq \pi \leq \pi_{0} \Rightarrow \beta(\sigma)(\pi)=\beta(\sigma)\left(\pi_{0}\right)$, so $(20)$ holds. Thus Serra's connective [23] and composed (multi-stage) [22] segmentations satisfy all segmentation requirements.

On the other hand, in Soille's constrained connective segmentation [27$30], \beta(\sigma)$ is an OC-thinning, so (20) does not necessarily hold. We saw for example in Figure 12 two partial partitions $\left\{X_{3}, X_{4}\right\}$ and $\mathbf{1}_{X_{2}}$ that satisfy $\beta(\sigma)\left(\mathbf{1}_{X_{2}}\right)<\beta(\sigma)^{2}\left(\left\{X_{3}, X_{4}\right\}\right)=\beta(\sigma)\left(\left\{X_{3}, X_{4}\right\}\right) \leq\left\{X_{3}, X_{4}\right\}<\mathbf{1}_{X_{2}}$, thus (20) is violated for $\pi_{0}=\mathbf{1}_{X_{2}}$ and $\pi=\beta(\sigma)\left(\left\{X_{3}, X_{4}\right\}\right)$. We could restrict the predicates used in order to obtain a C-thinning, but then this would give an opening. Indeed, given a predicate $\mathrm{p}$, if $\mathrm{B}\left(\tau_{p}\right)$ is a $\mathrm{C}$-thinning, then by Propositions 6,7 and $16, \tau_{\mathrm{p}}$ is an opening and $\mathrm{B}\left(\tau_{\mathrm{p}}\right) \mathrm{CS}^{\mathcal{C}}=\mathrm{CS}^{\mathcal{C}_{\mathrm{p}} \cap \mathcal{C}}$, where $\mathcal{C}_{\mathrm{p}}$ is a partial connection. Thus we fall back into block splitting openings, in other words Soille's approach reduces to Serra's in this case.

Although OC-thinnings do not satisfy (20), an interesting point is that they constitute a Moore family, while this is not the case for thinnings (antiextensive idempotent operators); overcondensation might well be the weakest possible property related to order, that guarantees the idempotence of a supremum of thinnings.

The concepts of C-thinning and OC-thinning were introduced by the author $[11,12]$ in order to describe the behaviour of some morphological operators on binary or grey-level images (i.e., sets and numerical functions) given in the literature. Afterwards these two concepts remained unused, until the author saw that the operators underlying Soille's segmentation approach [28] and the attribute thinnings on sets [4] (cf. Remark 19) are OC-thinnings, then that Serra's construction for multi-stage segmentation $[22,17]$ is the analogue, for partial partitions, of an old algorithm [10] for the decomposition of a set by a residual combination of set openings, and shares with it the property of being a C-thinning.

Due to lack of space, we have left out some special classes of operators. Soille [27] emphasized the importance of antitone predicates; indeed, they facilitate computations of operators like those of Corollary 15. Serra [24] showed 
also, in the framework of Corollary 15, the need for antitone predicates in order to guarantee the so-called "regional knowlege" in segmentation, namely to obtain, for any subset $B$ of a set $X$, a smallest possible set $B^{\prime}$ with $B \subset B^{\prime} \subseteq X$ such that $B^{\prime}$ determines if $B$ is a block of $\sigma(X)$. A link [20] is the meet of an isotone operator and an antitone operator. Now when a predicate $\mathrm{p}$ is antitone (more generally, when $\mathrm{p}$ is a link), it can be shown that $\tau_{\mathrm{p}}, \mathrm{B}\left(\tau_{\mathrm{p}}\right)$ and $\mathrm{B}\left(\tau_{\mathrm{p}}\right) \mathrm{CS}^{\mathcal{C}}$ are links. An operator $\psi$ is inf-separable [1] if for $x, y, z$ such that $x \leq y \leq z$, $\psi(x) \wedge \psi(z) \leq \psi(y)$. Every link is inf-separable, and in a frame (complete lattice where the binary meet distributes arbitrary suprema), reciprocally every inf-separable operator is a link. This equivalence applies in particular to predicates and trivial operators (because $\{0,1\}$ and $\mathcal{P}(E)$ are frames). However $\Pi^{*}(E)$ is not a frame, and indeed we give here a counter-example: let $E=\mathbf{Z}^{2}$, for any $p \in E$ write $\lambda(x)$ (resp., $\rho(x)$ ) for the point $q \in E$ that is the left (resp., right) neighbour of $p$, and for any $X \in \mathcal{P}(E)$, set

$$
\begin{aligned}
L(X) & =\{p \in X \mid \lambda(p) \notin X, \rho(p) \in X\} \\
\text { and } \quad & R(X)=\{p \in X \mid \lambda(p) \in X, \rho(p) \notin X\}
\end{aligned}
$$

(so $L(X) \cap R(X)=\emptyset$ ); now define the set splitting operator

$$
\sigma: \mathcal{P}(E) \rightarrow \Pi^{*}(E): X \mapsto \mathbf{1}_{L(X)} \vee \mathbf{1}_{R(X)} ;
$$

then $\sigma$ and $\beta(\sigma)$ are inf-separable but not links.

The connective segmentation approach $[23,17,13]$, as well as its composed (i.e., multi-stage) application [22,17] and its constrained variant [27-30], share common features. They associate to each function one or several partial connections, whose elements are generally built by chaining basic bricks called seeds [23,13], and then the segmentation is obtained by lattice-theoretical operations on partial partitions involving these seeds. The lattice-theoretical properties required from the various operations allow to take into account general knowledge about the meaning of the segmentation process (for example idempotence stands for the stability of the result, and isotony means that a join of objects not split or erased by segmentation will give an object that will also not be split or erased, cf. Figures 1 and 2). On the other hand, the choice of the criterion that associates to each function a partial connection (in practice, the extraction of seeds from the function) allows to take into account specific knowledge about objects to be segmented (for example in angiography $[8,9]$ : seeds are narrow and elongated connected bright zones surrounded by a darker image portion).

To conclude: we have studied idempotent anti-extensive operators on partial partitions, that are relevant in image segmentation methods based on the decomposition of space into homogeneous regions. They can also be applied in situations where the image segmentation produces a partition that is too coarse (this phenomenon is called under-segmentation). An opposite approach segments images by growing or clustering multiple seeds. This provides thus a rationale for investigating extensive operators on partial partitions, modelling such a process; they could also be applied in situations where the image 
segmentation produces a partition that is too fine (this phenomenon is called over-segmentation). Of particular interest are closures on partial partitions (for example by clustering blocks). Since the lattices $\Pi(E)$ and $\Pi^{*}(E)$ are not auto-dual, the theory of extensive operators should have no similarity to that of anti-extensive ones.

In our ongoing study of lattice-theoretical and monoid properties of maps on partial partitions, we have already analysed adjunctions on partitions and partial partitions [15]. Besides the study of extensive operators, such as block clustering ones, an interesting but more difficult problem is to apply to partial partitions well-known "topological" notions used in imaging sciences, such as connectivity, connected components and their reconstruction from seeds (i.e., the well-known flood-fill algorithm). Note in this respect the extension of the notion of connection to complete lattices $[21,16,3]$, and the first description of reconstruction operators on partitions [14].

Acknowledgements The author has for several years discussed with Jean Serra about connections, partitions and segmentation. More recently, he started such a dicussion with Pierre Soille. The presentation of our two papers benefited from the constructive suggestions by the referees and the editors.

\section{References}

1. Banon, G.J.F., Barrera, J.: Decomposition of mappings between complete lattices by mathematical morphology: Part I. General lattices. Signal Processing 30, 299-327 (1993)

2. Bloomberg, D., Maragos, P.: Generalized hit-miss operations. In: P. Gader (ed.) Image Algebra and Morphological Image Processing 1998, Proceedings, SPIE Proceedings, vol. 1350, pp. 116-128. SPIE, San Diego, California, USA (1990)

3. Braga-Neto, U., Goutsias, J.: Connectivity on complete lattices: new results. Computer Vision and Image Understanding 85(1), 22-53 (2002)

4. Breen, E.J., Jones, R.: Attribute openings, thinnings, and granulometries. Computer Vision and Image Understanding 64(3), 377-389 (1996)

5. Heijmans, H.: Morphological Image Operators. Advances in Electronics and Electron Physics Series. Academic Press, Boston (1994)

6. Heijmans, H.: Connected morphological operators for binary images. Computer Vision and Image Understanding 73(1), 99-120 (1999)

7. Matheron, G.: Random Sets and Integral Geometry. J. Wiley and Sons, New York (1975)

8. Naegel, B., Passat, N., Ronse, C.: Grey-level hit-or-miss transforms - Part I: Unified theory. Pattern Recognition 40(2), 635-647 (2007)

9. Naegel, B., Passat, N., Ronse, C.: Grey-level hit-or-miss transforms - Part II: Application to angiographic image processing. Pattern Recognition 40(2), 648-658 (2007)

10. Pitas, I., Venetsanopoulos, A.: Morphological shape decomposition. IEEE Transactions on Pattern Analysis and Machine Intelligence 12(1), 38-45 (1990)

11. Ronse, C.: Toggles of openings, and a new family of idempotent operators on partially ordered sets. Applicable Algebra in Engineering, Communication and Computing $\mathbf{3}$, 99-128 (1992)

12. Ronse, C.: A lattice-theoretical morphological view on template extraction in images. Journal of Visual Communication and Image Representation 7(3), 273-295 (1996)

13. Ronse, C.: Partial partitions, partial connections and connective segmentation. Journal of Mathematical Imaging and Vision 32(2), 97-125 (2008). DOI 10.1007/s10851-0080090-5 
14. Ronse, C.: Reconstructing masks from markers in non-distributive lattices. Applicable Algebra in Engineering, Communication and Computing 19(1), 51-85 (2008)

15. Ronse, C.: Adjunctions on the lattices of partitions and of partial partitions. Applicable Algebra in Engineering, Communication and Computing 21(5), 343-396 (2010). DOI $10.1007 / \mathrm{s} 00200-010-0129-\mathrm{x}$

16. Ronse, C., Serra, J.: Geodesy and connectivity in lattices. Fundamenta Informaticae 46(4), 349-395 (2001)

17. Ronse, C., Serra, J.: Fondements algébriques de la morphologie. In: L. Najman, H. Talbot (eds.) Morphologie Mathématique 1 - approches déterministes, chap. 2, pp. 49-96. Hermès / Lavoisier, Paris, France (2008)

18. Serra, J.: Image Analysis and Mathematical Morphology. Academic Press, London (1982)

19. Serra, J. (ed.): Image Analysis and Mathematical Morphology, II: Theoretical Advances. Academic Press, London (1988)

20. Serra, J.: The links: definitions and properties. In: M. Kunt (ed.) 5th Visual Communication and Image Processing Conference, SPIE Proceedings, vol. 1360, pp. 202-213. SPIE (1990)

21. Serra, J.: Connectivity on complete lattices. Journal of Mathematical Imaging and Vision 9(3), 231-251 (1998)

22. Serra, J.: Morphological segmentations of colour images. In: C. Ronse, L. Najman, E. Decencière (eds.) Mathematical Morphology: 40 Years On, Computational Imaging and Vision, vol. 30, pp. 151-176. Springer-Verlag, Dordrecht (2005)

23. Serra, J.: A lattice approach to image segmentation. Journal of Mathematical Imaging and Vision 24(1), 83-130 (2006)

24. Serra, J.: Consistency and regional knowledge in constrained connective segmentation. Tech. rep., ESIEE/A2SI/IGM (2009)

25. Serra, J., Vincent, L.: An overview of morphological filtering. Circuits, Systems and Signal Processing 11, 47-108 (1992)

26. Soille, P.: Morphological Image Analysis: Principles and Applications, 2nd edn. Springer, Berlin Heidelberg (2003)

27. Soille, P.: On genuine connectivity relations based on logical predicates. In: Proc. of 14th Int. Conf. on Image Analysis and Processing, Modena, Italy, pp. 487-492. IEEE Computer Society Press (2007)

28. Soille, P.: Constrained connectivity for hierarchical image partitioning and simplification. IEEE Transactions on Pattern Analysis and Machine Intelligence 30(7), 1132-1145 (2008)

29. Soille, P., Grazzini, J.: Advances in constrained connectivity. In: D. Coeurjolly, I. Sivignon, L. Tougne, F. Dupond (eds.) Discrete Geometry for Computer Imagery, LNCS, vol. 4992, pp. 423-433. Springer (2008)

30. Soille, P., Grazzini, J.: Constrained connectivity and transition regions. In: M. Wilkinson, J. Roerdink (eds.) International Symposium on Mathematical Morphology 2009, LNCS. Springer (2009) 